\title{
METODOLOGÍA MULTIVARIANTE PARA MEDICIÓN DE LA VULNERABILIDAD GLOBAL Contextos urbanos menores-zona de Esquilán-Cochabamba
}

\author{
Huáscar Bolívar Vallejo \\ Universidad Mayor de San Simón de Cochabamba-Bolivia (UMSS) \\ Instituto de Investigaciones de Arquitectura y Ciencias del Hábitat \\ Directora del IIACH: Dra. Arq. Graciela Landaeta Rodríguez \\ huasbol@yahoo.es
}

\section{RESUMEN}

La posibilidad de medición de la vulnerabilidad ha estado por mucho tiempo en la mesa de las discusiones científicas, exhibiendo un escenario dual que debate sobre su viabilidad y pertinencia o, por el contrario, su inviabilidad. No obstante lo que no entra en discusión es que de darse esa posibilidad los resultados traerían muchos beneficios en cuanto la reducción de los efectos e impactos por desastre, pues dejarían la posibilidad de visualizar posibles escenarios de riesgos futuros para las ciudades, que tendrían que ser resueltos a través de una planificación prospectiva basada en la reducción de la vulnerabilidad. En esa perspectiva, proponemos el desarrollo de un "método multivariante para la medición de la vulnerabilidad global" aplicado en un contexto urbano menor, con el propósito de lograr una diagnosis cercana de los problemas que hacen a su desarrollo, para posteriormente esbozar algunas líneas para el ejercicio de una planificación urbana prospectiva.

Palabras clave: Vulnerabilidad Global, Planificación Urbana, Riesgos de Desastre

\begin{abstract}
The possibility of measuring vulnerability has been at the table of scientific debates for a long time while displaying a dual scenario with regard to the discussing of its viability and relevancy or, on the contrary, its non-viability. However, what doesn't enter into discussion in the case of given this possibility is that the outcomes would bring many benefits such as the decrease of the effects and impacts caused by disasters since the chance of visualizing possible future risk scenarios for the cities would be left behind inasmuch as they would be resolved through a prospective planning based on reduction of vulnerability. In that perspective, we propound to work out a" multivariate method to measure global vulnerability" applied in a smaller urban context with the purpose of achieving a close diagnosis of the problems concerning the development in order to subsequently outline some guidelines for the exercise of a prospective urban planning.
\end{abstract}

Keywords: Global Vulnerability, Urban Planning, Disaster Risks 


\section{INTRODUCCIÓN}

Muchas ciudades en América Latina enfrentan serios problemas concernientes a sus bajos niveles de desarrollo relacionados en paralelo con sus elevados niveles de vulnerabilidad. En el afán de entender este fenómeno muchos gobiernos y autoridades en planificación se han enfocado históricamente en el tratamiento de las vulnerabilidades de orden físico, principalmente, bajo la óptica sesgada de que las debilidades de las ciudades y sus sociedades están subordinadas a las inclemencias de las amenazas, pensadas éstas en el rango de amenazas naturales. Con el tiempo esta forma de ver el problema ha ido cambiando desde la nueva concepción de la Gestión del Riesgo, basada fundamentalmente en entender a los fenómenos de desastre como "procesos" y no como resultados, ampliando la noción de la vulnerabilidad más allá de los aspectos físicos y entendiendo que las amenazas van más allá de los aspectos estrictamente naturales. En ello se ha visto la necesidad de valorar la vulnerabilidad en su concepción "global".

Sin embargo la posibilidad de valoración de la vulnerabilidad, o de medición en un sentido más positivo, ha estado por mucho tiempo en la mesa de las discusiones científicas, exhibiendo un escenario dual que debate sobre su viabilidad y pertinencia o, por el contrario, la inviabilidad de ese ejercicio. Enmarcados dentro el grupo de investigadores y cientistas sociales que aseveran la viabilidad y pertinencia de la medición de la vulnerabilidad, y basándonos también en investigaciones propias sobre la "transformación cualitativa de la vulnerabilidad (Bolívar, 2011)" y en el "método multivariante para la medición de la Vulnerabilidad Global (Bolívar, 2015)", el presente artículo pretende demostrar la viabilidad de la medición de la vulnerabilidad en su concepción "global" y, mas allá aún, verificar sus propósitos de uso prospectivo para un mejor ejercicio de la planificación urbana a largo plazo con reducción de la vulnerabilidad.

En ello, nuestro artículo se estructura en cuatro partes esenciales: una primera de carácter introductorio en la que se exponen los antecedentes problemáticos y justificativos de la investigación, así como los objetivos de la misma, la hipótesis que la sostiene y el abordaje metodológico empleado; una segunda que aborda el escenario problemático de estudio (Esquilán y su relación con el contexto metropolitano) desde la perspectiva histórica alusiva a los desastres y la vulnerabilidad; una tercera en la que se presenta el método multivariante y se define la caracterización, medición y diagnóstico de la Vulnerabilidad Global de la zona de estudio a través de la aplicación y verificación del método multivariante; y una cuarta y última parte destinada a las reflexiones y conclusiones finales.

\section{PRESENTACIÓN DEL PROBLEMA Y JUSTIFICATIVOS DE LA INVESTIGACIÓN}

En el entendido que convencionalmente se asimila a los riesgos de desastre y los propios desastres como el producto de la "amenaza" por la "vulnerabilidad" y que en la mayoría de los casos no es posible trabajar sobre las amenazas -especialmente cuando éstas son de origen natural-, estudios recientes coinciden en que en América Latina la vulnerabilidad es un factor clave no resuelto en esa ecuación y profundizan que la vulnerabilidad en sí es precisamente un conjunto de vulnerabilidades conexas que interactúan entre sí y que su debilitamiento general proviene en su gran mayoría de la desarticulación de esas vulnerabilidades consideradas en el rango de factores. Esos factores que hace referencia la vulnerabilidad global son (con base en la propuesta de Wilches-Chaux, 1993; 1998 y 2007): el físico-estructural, el económico, el ecológico-ambiental, el técnico y el social, consignándole a este último los factores político- institucional, educativo, ideológico-cultural y relativos a la organización social.

A pesar que en muchas instancias de gestión existe plena conciencia de este problema, buena parte de los países en vías de desarrollo hacen pocos esfuerzos en reducir los estados de vulnerabilidad en cada factor y mucho menos esfuerzo aún en articular estos factores de manera fluida, interconectada y equilibrada. Ahondando más en el problema, existe también al interior de las instancias de gestión, y en un grueso grupo de la comunidad científica, el empeño por señalar que la medición de la vulnerabilidad no es pertinente debido a su estado efímero, cambiante y susceptible a interpretaciones disímiles por su relación con la intensidad oscilatoria del riesgo, pues este último es también susceptible a percepciones disímiles precisamente por la "vulnerabilidad diferenciada" que expone la sociedad heterogéneamente diversa ante la adversidad.

En ese sentido, muchos investigadores han manifestado su preocupación sobre la pertinencia o no de medir la vulnerabilidad desde el ámbito de los desastres, sobre su utilidad y sobre si ésta puede ser 
verdaderamente cuantificable (Cardona, 2007). Desde una postura personal y basados en la experiencia de posibilidades que la propia teoría nos sugiere, consideramos que la medición de la vulnerabilidad es posible y pertinente con fines diagnósticos, de seguimiento y monitoreo, de manera que esta práctica permita la toma de decisiones más acertadas en el ejercicio de la planificación urbana.

En anteriores investigaciones habíamos introducido un nuevo concepto: "la transformación cualitativa de la vulnerabilidad" (Bolívar, 2011; 2015). Con este concepto complejizamos aún más el escenario de la cuantificación de la vulnerabilidad, pues consideramos que ésta era susceptible a no sólo ser medida en un corte temporal definido y sólo sobre variables conocidas en ese momento, sino también a ser medida en la dimensión espacio tiempo y sus posibles mutaciones. Asumimos a la "dimensión cualitativa" como aquella parte de la vulnerabilidad que, además de ser distinta a través del tiempo, es capaz de mutar a un rango de vulnerabilidad desde un estado anterior en el cual podría no haber sido considerada como vulnerabilidad, o no haber sido percibida como tal. En forma más explícita, la dimensión cualitativa de la vulnerabilidad vendría a ser aquel espacio-tiempo en el que lo que no es considerado como vulnerabilidad o indefensión en un momento, pasa a convertirse en vulnerabilidad con nuevas características que la definen como vulnerabilidad transformada. Lo "cualitativo" en la vulnerabilidad sería aquello capaz de involucrar un cambio en su estructura, aquello que se transforma al momento en que aparecen en la vulnerabilidad nuevas características que la definen como distinta a través del tiempo; en otras palabras: nuevas variables cualicuantitativas de la vulnerabilidad.

Si bien es complejo el escenario de la mensura de la vulnerabilidad, es importante considerar que su medición, o valoración, resulta imprescindible a la hora de establecer los niveles de riesgo en la ecuación $($ Riesgo = Amenaza $\times$ Vulnerabilidad), mucho más cuando ese riesgo implica la posible evolución de su estado al "desastre" mismo. Por lo tanto, arrimándonos a la posición de Omar Darío Cardona (2007), la medición de la vulnerabilidad con fines de "proxies" o seguimiento permitiría a las instancias de gestión y toma de decisiones diseñar planes, programas y proyectos con visiones más sostenibles, puesto que, en la lógica de una sociedad en desarrollo, trabajar en la reducción de la vulnerabilidad (a través de inversiones para la reducción de la misma) es un sinónimo de trabajar en pos de la sostenibilidad. A todo ello, valdría la pena preguntarse: ¿Cómo es posible reducir los niveles nocivos en un factor de la vulnerabilidad sino sabemos cuanto mide, equivale o el peso que tiene? En otras palabras: ¿Cómo podemos realizar una gestión del riesgo efectiva si no podemos medir (diagnosticar) la vulnerabilidad? Y ¿Cómo podemos pretender reducir el riesgo si no sabemos el grado de vulnerabilidad que debemos reducir? Estas preguntas justifican con creces la necesidad de profundizar más sobre la pertinencia de la mesura de la vulnerabilidad.

En ese sentido, avanzando sobre nuestras investigaciones acerca del estado del conocimiento científico entorno a la vulnerabilidad global, introducimos en este artículo la aplicación del método multi-variante propuesto para verificar el estado y tipo de relacionamiento de los factores de la Vulnerabilidad Global.

\section{OBJETIVOS}

El artículo que se propone tiene por objetivo principal diagnosticar la vulnerabilidad global manifestada en la zona de Esquilán a través del método multivariante, con el propósito de delinear criterios prospectivos para un mejor ejercicio de la planificación urbana a largo plazo con reducción de la vulnerabilidad.

Paralelamente se plantean cuatro objetivos específicos: (1) Identificar estratégicamente sucesos de interés relacionados a problemas de desastres en Esquilán en una línea de tiempo de 20 años, comprendida entre 1992 y 2012; (2) Caracterizar los factores de la Vulnerabilidad Global presentes en la zona; (3) Diagnosticar el estado de la Vulnerabilidad Global e (4) identificar los caminos dinámicos que podrían seguir a futuro los diversos factores de la V.G. en el esquema espacio-tiempo.

\section{METOdOLOGÍA}

El desarrollo de nuestros objetivos fue acompañado por la adopción de métodos cualitativos y cuantitativos, debido al carácter múltiple planteado tanto en el problema como en los justificativos del proyecto.

El desarrollo del primer objetivo específico necesitó de una minuciosa revisión bibliográfica históricahemerográfica y actualizada, así como de la encuesta estructurada a pobladores de la zona e instancias 
pertinentes de gestión y planificación. Por su parte el segundo objetivo específico se convirtió funcionalmente en el punto de relación entre el primero y los últimos dos. La información básica conseguida en el segundo objetivo provino de la aplicación de técnicas de campo, el relevamiento, la sistematización de patrones y entrevistas a personajes y grupos clave.

La investigación a partir del segundo objetivo específico tuvo un carácter esencialmente cuantitativo y adoptó la sistematización de la información para generar metodológicamente la identificación de indicadores de cada factor de la vulnerabilidad; el monitoreo de datos verificables en base a estadísticas, datos significativos, recuentos y otros, se constituyeron en las técnicas aplicadas para este fin.

Desde una perspectiva general, nuestra investigación fue esencialmente de modalidad "empírica", siendo caracterizada por la descripción del escenario de estudio en sus facetas situacionales y causales, por lo que se planteó un tipo de investigación "descriptiva" para el primer objetivo, ya que abordó el problema desde la descripción de una realidad a partir de los esquemas conceptuales de la "Vulnerabilidad Global", y de tipo "explicativa-analítica" para los objetivos posteriores, porque estableció relaciones causales y de interconexión entre los diversos factores de la vulnerabilidad global.

\section{HIPÓTESIS}

Desde el enfoque de nuestra investigación, se plantea que la Vulnerabilidad es susceptible a incrementar, mantener o reducir sus niveles en tiempo y espacio, empero, en una suerte de cambio dinámico, es susceptible también a transformarse cualitativamente en su propia estructura dentro la dimensión espaciotiempo, al punto de derivar no sólo en la variación de niveles diversos cuantificables, sino en la mutación de nuevas formas cualitativamente dinámicas susceptibles también a su cuantificación. En este artículo nuestra hipótesis de trabajo asevera que la valoración y reducción de la vulnerabilidad son posibles a través de la medición y nivelación en positivo de cada uno de los factores que componen la Vulnerabilidad Global, dentro las consideraciones metodológicas de "Tamaño", "Ubicación", "Longitud", "Resistencia" y "Conexión", propias del método multivariante propuesto.

\section{CASO DE ESTUDIO}

El escenario urbano menor elegido para la puesta en marcha del método multivariante se constituye en la zona de Esquilán, perteneciente al municipio de Colcapirhua (Cochabamba-Bolivia), municipio desarrollado dentro el eje de conurbación de la RMK, entre las ciudades de Quillacollo y Cercado desde el sector de Coña Coña (Km. 5,5) hasta el de Piñami (Km. 10), corresponde jurisdiccionalmente a la 5ta. Sección de la provincia de Quillacollo y pertenece a la unidad fisiográfica y subregión del "Valle Central de Cochabamba". Según datos del PDM (2005-2009) y el PLANUR (2003-2013), insertos en el "Diagnóstico Integral Participativo del Municipio de Colcapirhua (2009), está entre los paralelos $17^{\circ} 21^{\prime} 20^{\prime \prime}$ y el $17^{\circ} 25^{\prime} 30^{\prime \prime}$ de

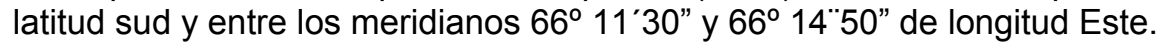

La extensión territorial de Colcapirhua es de $32,10 \mathrm{Km} 2$, estructurándose en 5 Distritos administrativos (A, $\mathrm{B}, \mathrm{C}, \mathrm{D}$ y $\mathrm{E}$ ), considerándose los Distritos $\mathrm{B}, \mathrm{C}$ y $\mathrm{D}$ como urbanos y los Distritos A (al norte) y $\mathrm{E}$ (al sur) como rurales; este último es el Distrito que alberga a Esquilán, con una extensión territorial promedio de $32,10 \mathrm{Km} 2$. Debido a su condición rural y las paradojas urbanas que suceden en él es que se suscitan muchos problemas ambientales y de riesgos no resueltos, habiéndose registrado múltiples sucesos de desastres asociados a la amenaza de la informalidad urbana, a la descoordinación administrativa, a la ausencia de planificación y a la debilidad y descoordinación de otros factores de la Vulnerabilidad Global.

\section{EL ESCENARIO PROBLEMÁTICO DE LA METRÓPOLI KANATA Y LA ZONA DE ESQUILÁN}

A partir de 1990, la embrionaria Región Metropolitana de Kanata ${ }^{1}$ sufrió severos efectos e impactos por el desencadenamiento de fenómenos naturales y no es de extrañar que las zonas con mayor vulnerabilidad hayan sido las que mayores daños presentaron ante esas situaciones.

\footnotetext{
${ }^{1}$ Metrópoli Kanata, recientemente nombrada así por Ley 533 de 27 de Marzo de 2014 (Ex metrópoli de Cochabamba, y desde nuestro punto de vista todavía embrionaria región metropolitana), con una población de 1.141.094 habitantes en 2012 (INE, 2012), se encuentra en la actualidad compuesta por 7 municipios (Sacaba, Cercado, Colcapirhua, Quillacollo, Vinto, Sipe-Sipe y Tiquipaya, llegando a sumar un total aproximado de $34 \mathrm{~km}$ lineales de extremo a extremo).
} 
Es importante resaltar que a medida que la metrópoli creció también se intensificaron los sucesos de desastre. La densificación produjo que los elementos vulnerables expuestos sean mayores, a tiempo de generar también mayor "vulnerabilidad física" (sociedad-territorio) debido a las transformaciones que se realizaron en el contexto inmediato, con capacidad de producir amenazas y riesgos en cadena. Con el paso del tiempo los sucesos de desastre sucedieron con mayor frecuencia y muchos de ellos se repitieron en un mismo lugar, lo que dice mucho sobre una "vulnerabilidad incremental", "baja resistencia" y, sobre todo, "baja resiliencia". Este fenómeno se debió principalmente a que muchas de las urbanizaciones que en la actualidad son consideradas en el rango de "formales" se iniciaron en el rango de "informales", en zonas despobladas con riesgo de inundaciones y desbordes, situación que en la actualidad puede esbozar un escenario urbano en el que los aspectos relacionados a la tenencia y la propiedad (de tipo dominial, en alusión a Clichevsky, 2000) han sido resueltos y no así los aspectos urbanos relacionados a los la gestión del riesgo propiamente.

Las diferencias en los pesos poblacionales y económicos en los distintos municipios jugaron un papel preponderante en la cantidad de los desastres suscitados en cada uno de ellos y en la dureza de los efectos e impactos por desastre. Veamos los gráficos 1 y 2 :

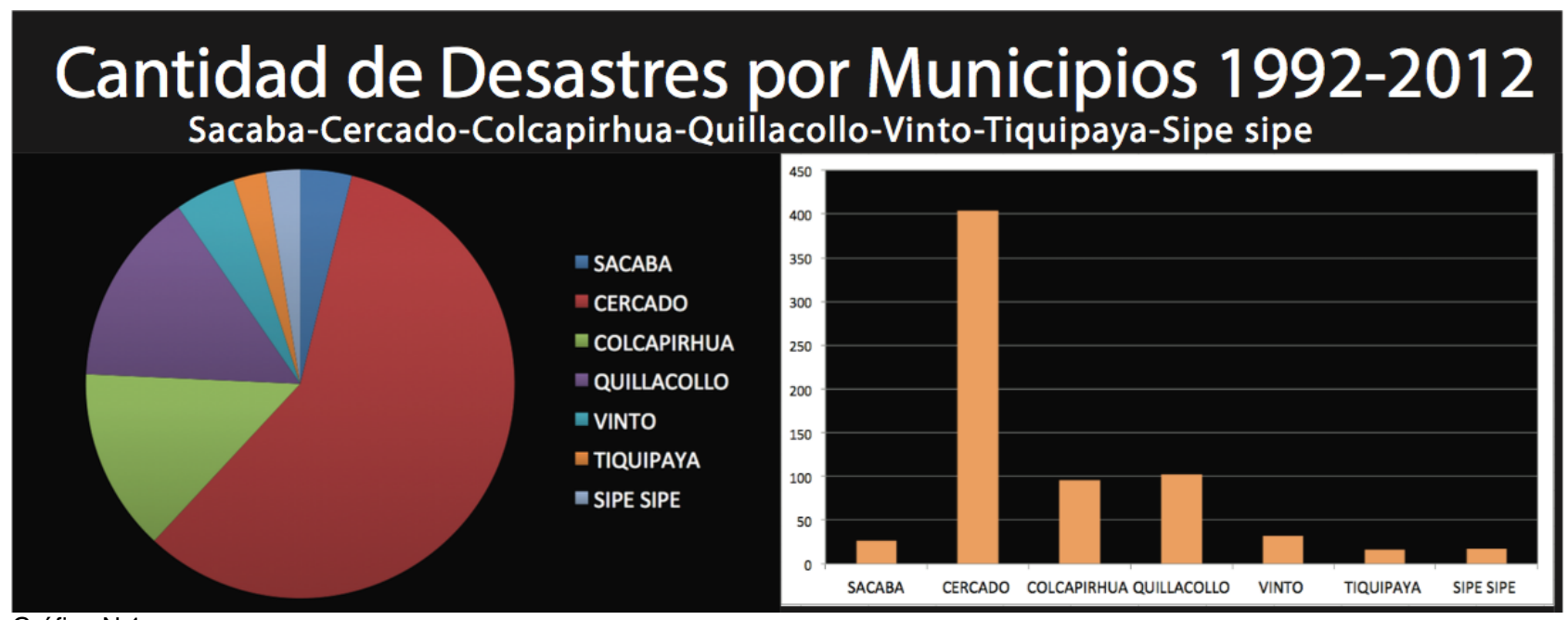

Gráfico N 1

Cantidad de desastres suscitados en la Metrópoli de Kanata entre 1992 y 2012

Elaboración propia con base en Bolívar 2011, 2015.

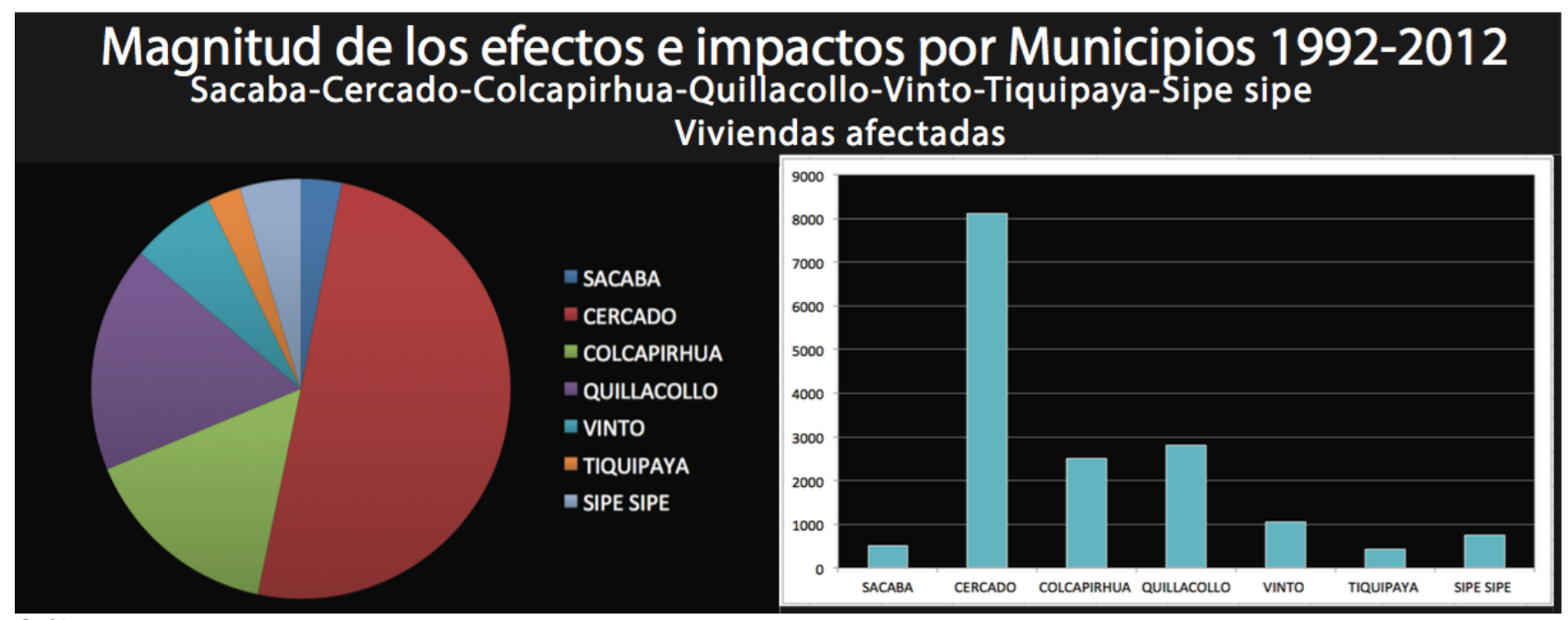

Gráfico N 2.

Efectos que corresponden a la cantidad de viviendas afectadas entre 1992 y 2012 Elaboración propia con base en Bolívar 2011, 2015. 
Apelando a una modelación del embrionario escenario de principio del siglo $\mathrm{XX}$, de lo que hoy se conoce como la metrópoli Kanata, y haciendo una relación de lo que fueron los sucesos de desastre de origen hidrometeorológico en el periodo 1992-2012, se puede observar que la gran mayoría de éstos se dieron fuera de lo que la mancha urbana representó a principio de 1900 (nodos dispersos). Esto refuerza tres planteamientos fundamentales: (1) que los desastres se incrementaron a medida que la mancha urbana creció, (2) que no existió una planificación Ex ante y oportuna para el desarrollo urbano de lo que se define como "metrópoli Kanata" y (3) que los sucesos de desastre se consideraron como tales porque los efectos e impactos de los fenómenos hidrometeorológicos se manifestaron sobre elementos vulnerables expuestos y no sobre campos de cultivos o áreas rústicas como sucedió en el pasado.

Veamos gráficamente esa situación:

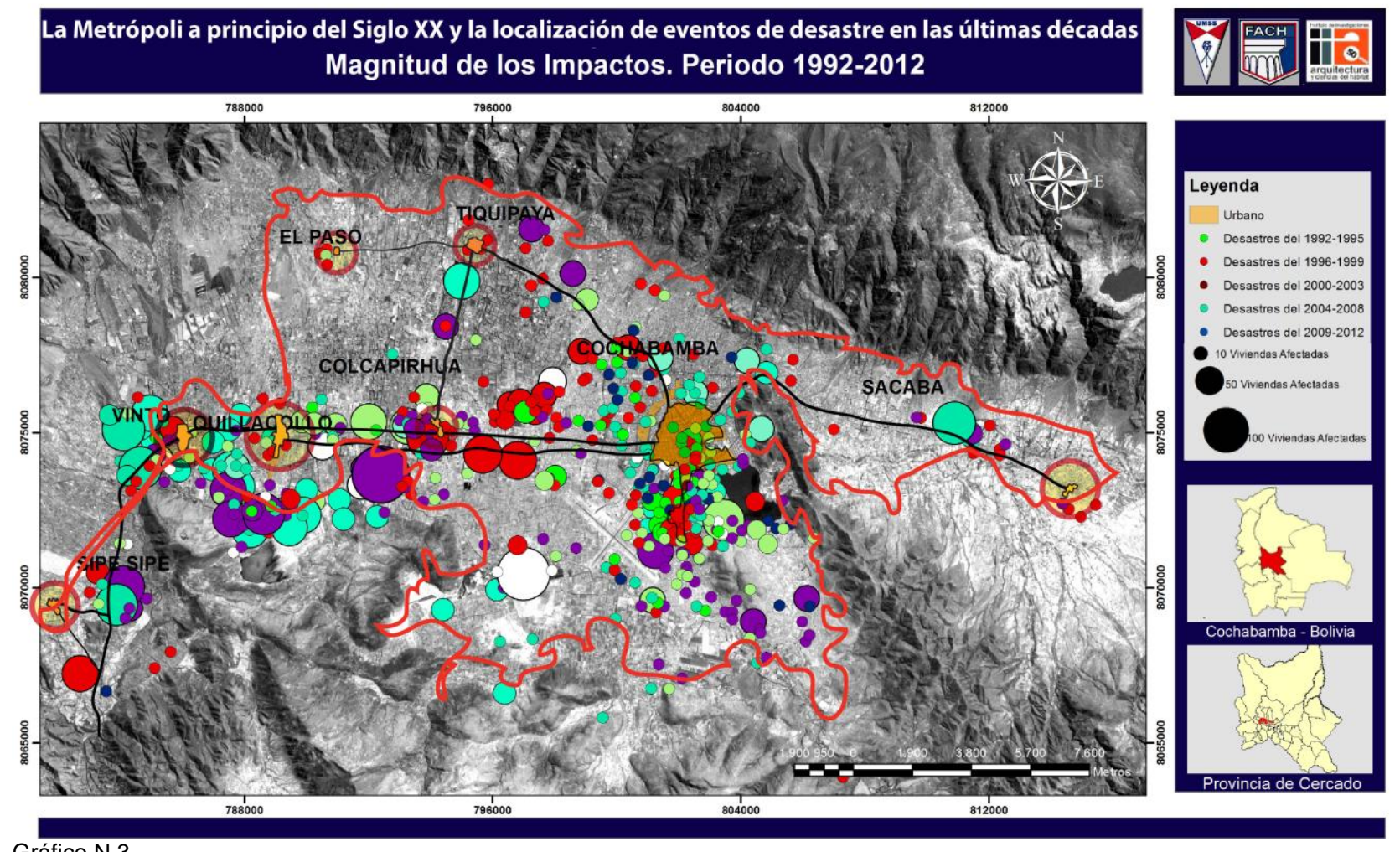

Gráfico N 3.

Eventos de desastre expresados en mapas simultáneos 1900 y 2016

Nodos urbanos de principio de Siglo XX y Borde metropolitano 2016

Elaboración propia con base en información geográfica de PROGEO (Programa de Geografía IIACH-UMSS).

\subsection{El caso de Esquilán-Colcapirhua}

Entrando en tema, la zona de Esquilán es un ejemplo recurrente de problemas de desastre que se relacionan con la descoordinación administrativa y la ausencia de planificación prospectiva a nivel metropolitano, además es también un ejemplo recurrente de debilidades en cuanto al control municipal del uso del suelo y problemas ambientales, a lo que habría que agregar la debilidad de otros factores de la Vulnerabilidad Global sobre los cuales no se ejercen acciones de hecho, como el educativo y el cultural en temas relacionados a los riesgos; de ahí la pertinencia de su estudio como modelo de implementación del método multi-variante.

A lo largo de estos últimos años se han registrado en esta zona numerosos casos de desastres, caracterizados por inundaciones y anegamientos con la pérdida y destrozo de viviendas por desbordes de los ríos Rocha, Pampa Mayu, Chijllawiri y Tamborada en lo que corresponde al aspecto físico-estructural, así como la alteración negativa de muchos otros factores de la Vulnerabilidad Global, como el ambiental, el económico, el institucional, el social y otros.

A continuación se presenta una lista de eventos de desastres de origen hidro-meteorológico y la magnitud de estos en la zona de Esquilán: 
- En 1997 fueron más de 20 de viviendas afectadas

- En 2000 más de 30 viviendas afectadas

- En 2001 se repitieron en 4 oportunidades las inundaciones ese mismo año y fueron más de 70 viviendas afectadas y varias perdidas completamente.

- En 2006 se repitieron en 2 oportunidades las inundaciones y fueron más 60 viviendas afectadas. Barrios vecinos en Sumumpaya y Santo Domingo también se inindaron.

- En 2007 se repitieron en 3 oportunidades las inundaciones y fueron más de 40 viviendas afectadas.

- En 2008. En el caso de Colcapirhua (y parte del Cercado), las zonas de Sumumpaya y Esquilán Grande fueron las más afectadas con el violento desborde de los ríos Rocha (alimentado por el río Tamborada), Pampa Mayu y Chijllahuiri el 3 de febrero de 2008, dejando un saldo de 300 familias damnificadas, muchas de las cuales perdieron su vivienda. En el barrio de Esquilán Grande, de 86 familias afectadas fueron 32 que se quedaron sin vivienda. Luego del suceso, quedaron por varias semanas alrededor de 40 familias viviendo en carpas precarias.

- En 2009 fueron más de 30 viviendas afectadas por inundación y muchas vías quedaron anegadas e intransitables por varios días.

- En 2010 fueron más de 25 viviendas afectadas por inundación

- En 2011 más de 25 viviendas afectadas por inundación

- Por último en 2012 (fecha hasta la cual producimos esta información) fueron más de 20 viviendas afectadas por inundación.

Observemos gráficamente esa situación:

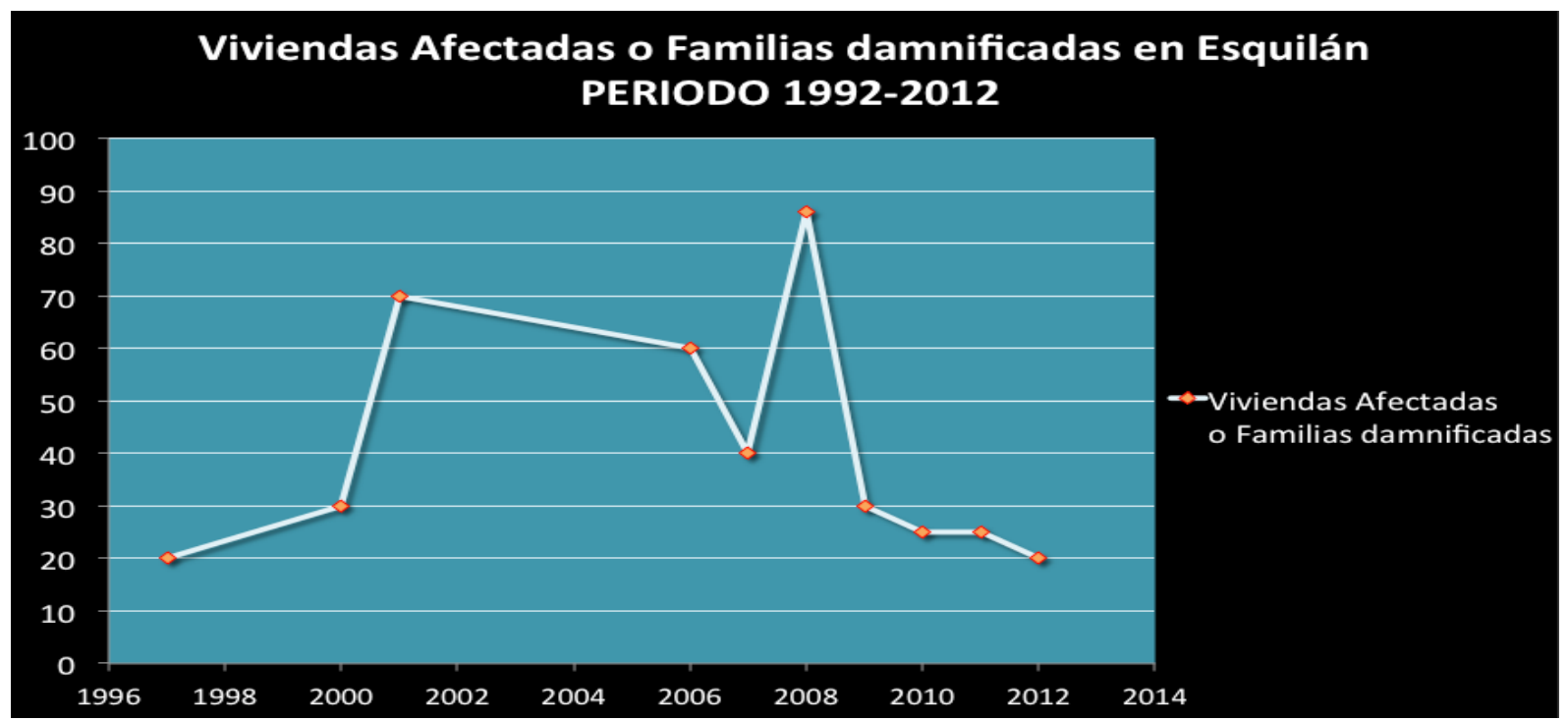

Gráfico N 4

Cantidad de viviendas afectadas en Esquilán durante el periodo 1992-2012

Fuente: Elaboración propia con base en Bolívar 2011, 2016.

Una vez sucedidos los desastres en Esquilán, los procesos históricos de estos acontecimientos evidenciaron las lentas y descontextualizadas acciones institucionales (muchas veces de intervención parcial en el problema, como también inconclusas) que no permitieron el restablecimiento de la comunidad por lo menos a su estado anterior en el corto plazo, manifestando fallas en el factor institucional de la vulnerabilidad. Por otra parte, estos sucesos dejaron también al descubierto la debilidad del factor político en cuanto al escuálido compromiso preventivo y prospectivo por reducir la vulnerabilidad en el momento oportuno, es decir, antes que se presenten los fenómenos. La idea de que "lo peor ya pasó" y que el desastre no se volverá a presentar fue el ingenuo consuelo desde la óptica cultural que no permitió reinterpretar los nuevos estados de la vulnerabilidad, siendo la experiencia del suceso tan sólo el medio para verificar estados de la vulnerabilidad al fenómeno presentado y no así para reinterpretar los riesgosos escenarios futuros, raramente vislumbrados cuando el enfoque de la "emergencia" es el que predomina en el tiempo presente. Por otra parte, la ausencia de programas e instrucción básica en gestión de riesgos desnudó altas debilidades en al factor educativo a diferentes escalas. Todo ello, en combinación con el debilitamiento del factor económico por las pérdidas luego del desastre y por el uso de recursos económicos no siempre acertados destinados sólo en las acciones para el restablecimiento de las 
condiciones físicas y ambientales (algunas), no han hecho otra cosa que incrementar la vulnerabilidad y transformarla a pasos más veloces que las pasivas acciones en prevención.

A pesar de haberse registrado algunas inversiones económicas de parte del municipio en los últimos años, dirigidas principalmente al ensanche y pavimentado de algunas calles, a canalizaciones y a otros servicios básicos a nivel parcial, la zona de Esquilán presenta en la actualidad un ambiente insalubre, donde los malos olores y la mala calidad del aire son permanentes, donde el recurso suelo es extremadamente frágil por el consumo excesivo de material por la industria ladrillera (49 en total) y por la propia urbanización informal que se provee de los recursos del entorno para la construcción y consumo energético. En ello, Esquilán tampoco se libra de elementos tanto internos (fabricas de ladrillo, los ríos contaminados, etc.) como externos (contaminación atmosférica de la Av. Blanco Galindo, aguas servidas de barrios vecinos, a manera de ejemplo) que afectan a la población en términos de salubridad y seguridad física. Y si bien las situaciones de desastre son mucho más sentidas de manera puntual cuando los problemas hidrológicos se presentan, es obvio que esta zona presenta permanentemente otros problemas de desastre, entre pequeños y medianos, que deben hacerse visibles y ser contextualizados y analizados desde la perspectiva global.

\section{FACETAS MÁS IMPORTANTES DEL MÉTODO MULTI-VARIANTE}

Comencemos por indicar que la V.G. -propuesta por Gustavo Wilches-Chaux ya en 1993 en representación de la RED²- está compuesta por las siguientes Dimensiones: física-estructural, económica, ecológicaambiental, técnica y social, consignándole a esta última las dimensiones política-institucional, educativa, ideológica-cultural y relativas a la organización social:

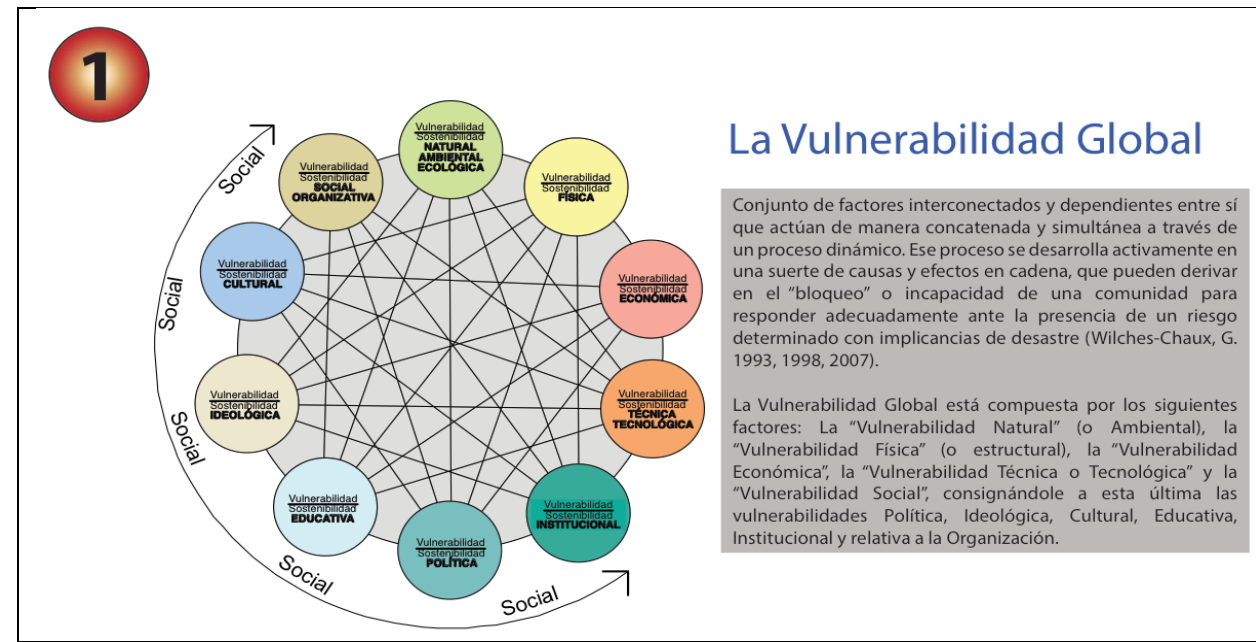

La Vulnerabilidad es Global y su Estado depende de la debilidad o fortaleza de cada uno de sus factores o dimensiones, así como de la calidad de sus relaciones.

Gráfico N 5

\footnotetext{
${ }^{2}$ LA RED: Red de Estudios Sociales en Prevención de Desastres en América Latina. Creada en 1992 y compuesta por varios intelectuales e instituciones de renombre con sede en distintos países de América Latina, entre los que se encuentran Gustavo Wilches-Chaux, Omar Darío Cardona, Allan Lavell y Andrew Maskrey como sus máximos representantes.
} 
No obstante, la propia definición de "Vulnerabilidad Global" hace alusión a un estado de debilidad, por lo tanto es lógico entender que, además del estado de cada factor, éstos no siempre se encuentran bien relacionados, 0 que su relación puede ser incluso nula.
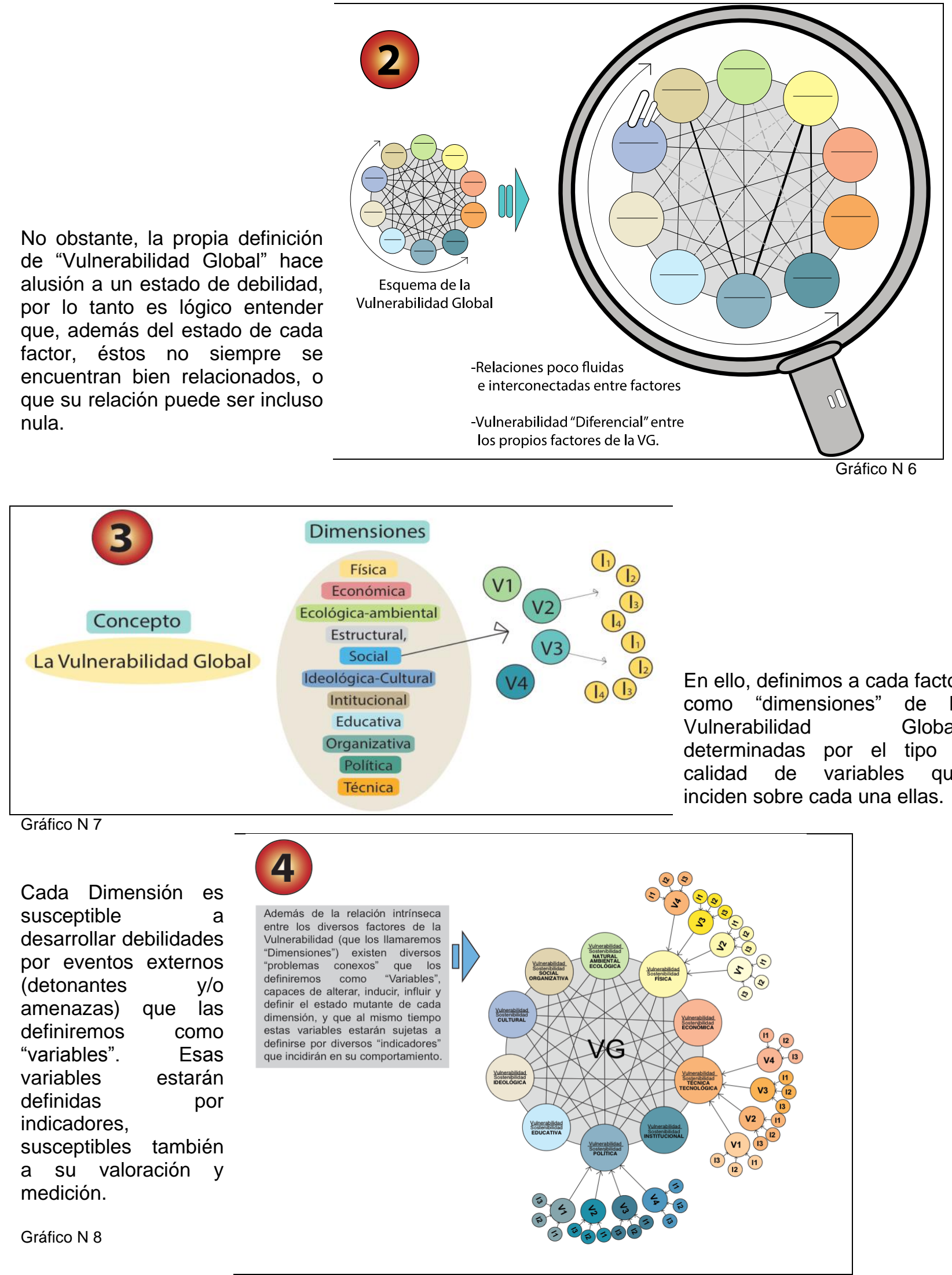

Un grueso grupo de la comunidad científica ha concertado en asimilar la sólida propuesta de Wilches-Chaux (2007) sobre la Vulnerabilidad Global en analogía a una "Telaraña"; esquema al cual nos sumamos y del cual emprendemos nuestra propuesta. 


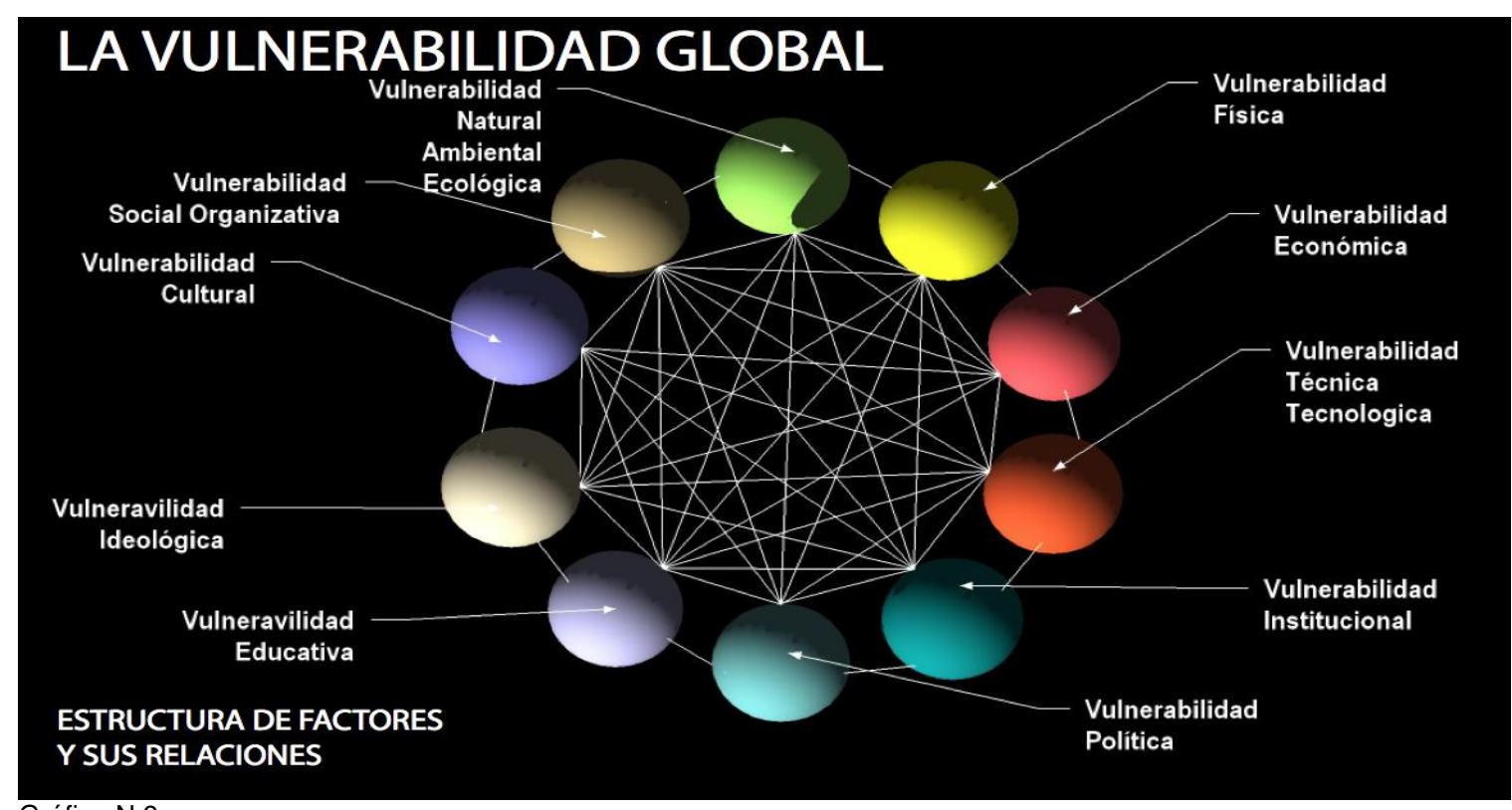

Gráfico N 9

Telaraña de la Vulnerabilidad Global

Elaboración propia con base en Gustavo Wilches-Chaux (2007)

El planteamiento de Wilches-Chaux fue esquematizado bidimensionalmente para entender el estado de la Vulnerabilidad Global en un momento del tiempo y desde la consideración conceptual que asume el estado de todos los factores y sus niveles de relación intrínseca. Sin embargo desde nuestra postura aportamos un poco más: consideramos que un recorte "Iongitudinal" en la línea del tiempo en realidad desnuda la vulnerabilidad diferencial entre cada uno de los factores. En ese corte se puede evidenciar que cada uno de los factores se encuentra en realidad desfasado; desfase que hace alusión a la calidad de acciones (positivas o negativas) para la reducción de la vulnerabilidad en cada factor. Veamos gráficamente esa situación analógica:

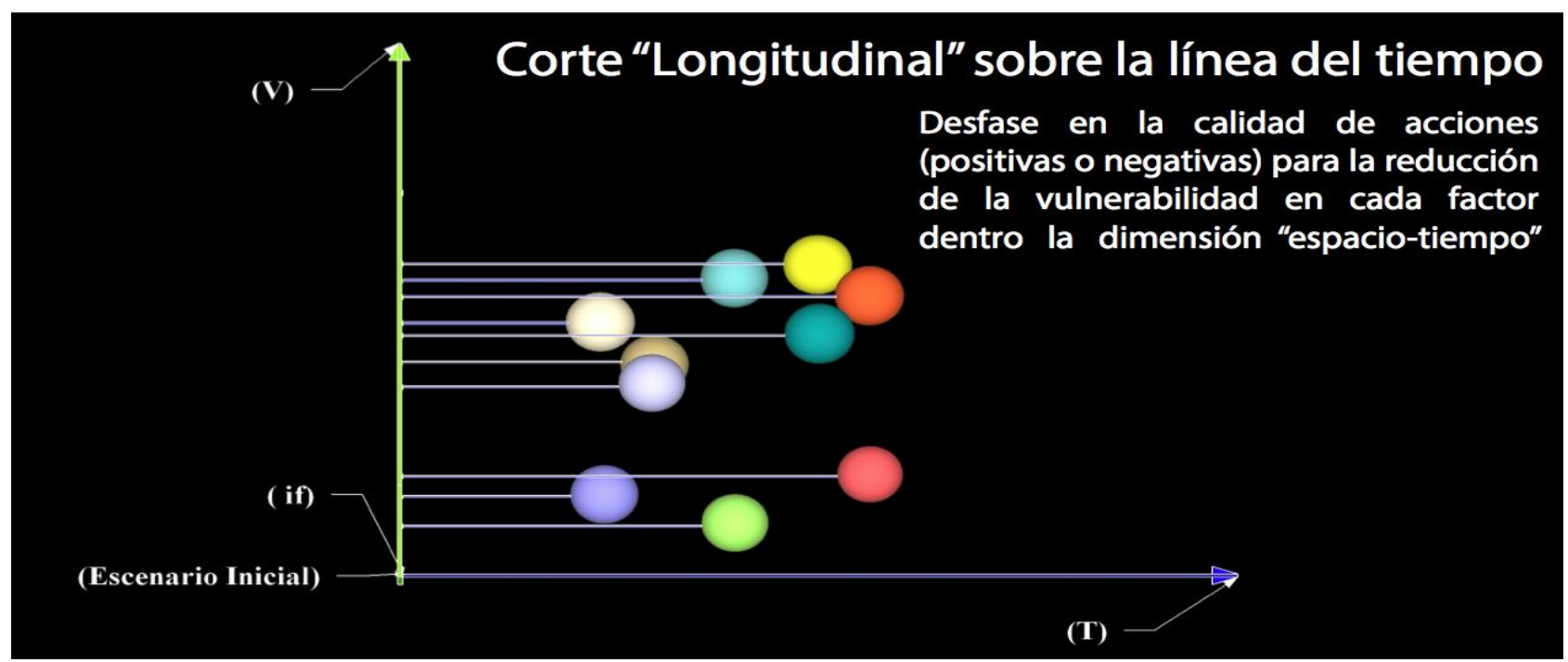

Gráfico N 10

Desfase de los factores de la V.G. en la dimensión espacio-tiempo Elaboración propia

Entonces, ¿Qué es lo que sucede en realidad con la "Telaraña" de la Vulnerabilidad Global en la dimensión espacio tiempo? Hagamos, paso a paso, un giro en perspectiva. Veamos poco a poco cuál es la imagen que obtendríamos si observamos de manera oblicua la situación de la Vulnerabilidad Global en la dimensión espacio-tiempo: 

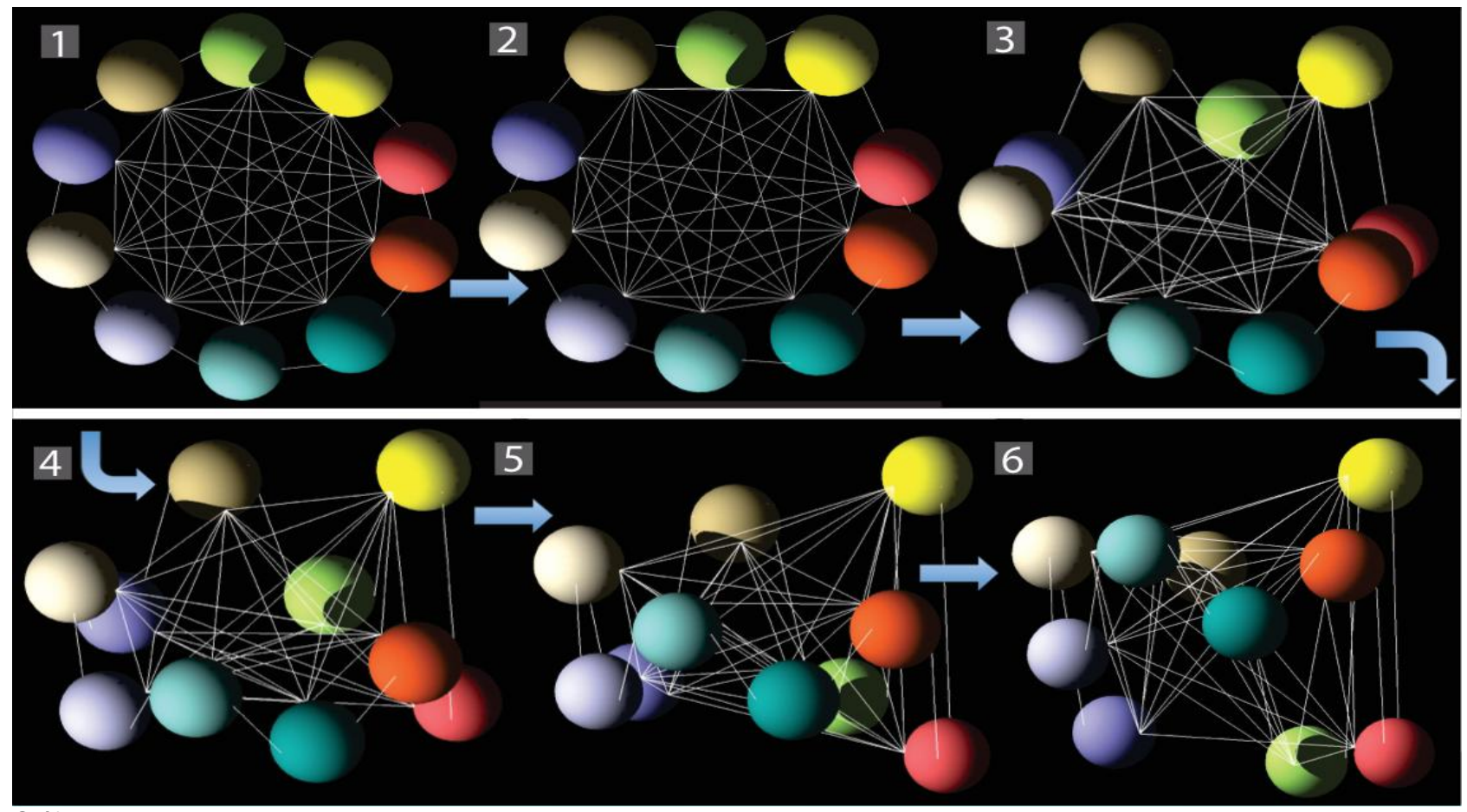

Gráfico N 11

Desfase de los factores de la V.G. en la dimensión Espacio-Tiempo

Elaboración propia

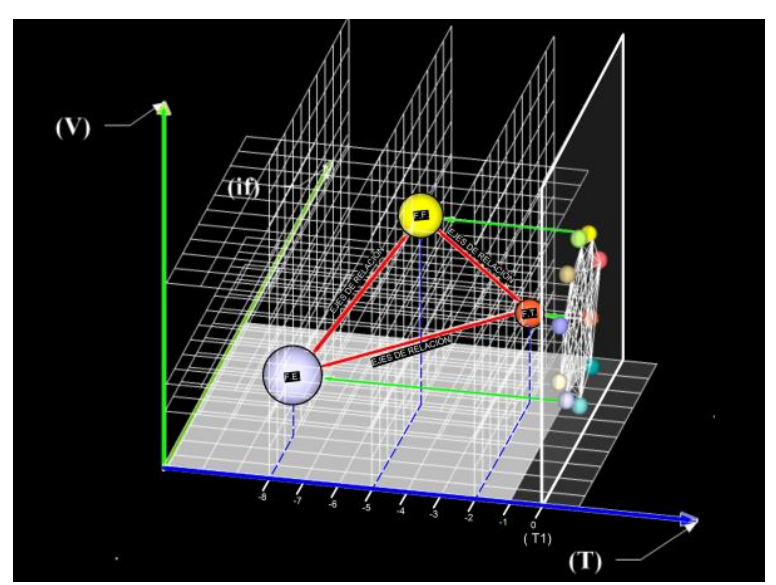

Las líneas de interconexión o relacionamiento que debieran conformar la telaraña en realidad no se llegan a tocar. Lo que debería conformar un conjunto de factores fortalecidos, por el contrario, expone que los factores se encuentran plenamente desfasados y con niveles diferenciados en cuanto a su vulnerabilidad. Por otra parte, lo que debería conformar una telaraña de conexiones fortalecidas a través de los puntos de intersección entre cada línea de relacionamiento no existe en realidad, precisamente por esos desfases en las acciones disímiles con relación al fortalecimiento de cada factor; lo que nos dice sobre la vulnerabilidad diferenciada entre los factores de la V.G.

Gráfico N 12

Distanciamiento y desconexión entre las líneas de relacionamiento entre factores de la V.G.

Elaboración propia

De todo ello, entonces, ¿Cómo se vería la telaraña? o, dicho de otra forma, ¿Cuál sería la condición espacial de la telaraña en la línea del tiempo?: 


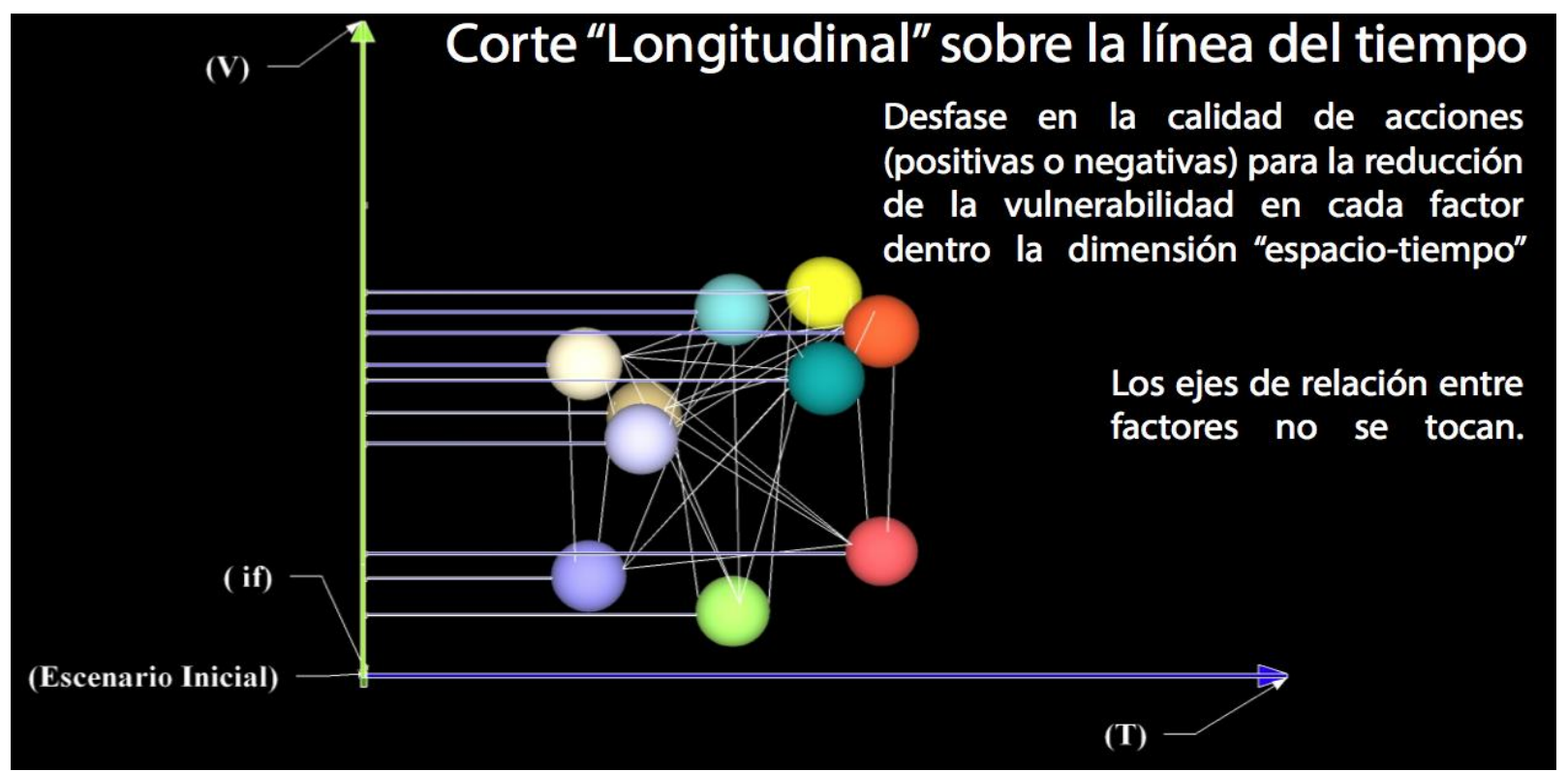

Gráfico N 13

Desfase de las Dimensiones de la V.G. sobre la línea del tiempo

Elaboración propia

\subsection{Base secuencial para el planteamiento del método multi-variante}

Algunos de los factores de la Vulnerabilidad Global pueden ser reducidos en el tiempo, otros mantenerse y otros incrementarse, lo que definiría al esquema tridimensional propuesto como un esquema dinámico en la dimensión espacio-tiempo. La clave, entonces, para la valoración de la Vulnerabilidad Global resulta ser la medición de ese estado tridimensional en el espacio-tiempo, donde:

1) la telaraña conceptual bidimensional deja de ser tal, al no encontrar intersecciones entre las relaciones que la componen,

2) el "tamaño" de cada factor determina asimismo un nivel de fortalecimiento o debilitamiento. Cuanto más grande es, mayor es la vulnerabilidad que representa.

3) la "ubicación" en espacio-tiempo de cada uno de los factores determina cualitativa y cuantitativamente las calidad de las acciones en favor de la resistencia y resiliencia que se ejercen sobre cada factor o dimensión de la V.G.. Cuanto más atrás está, significa mayor vulnerabilidad y se encuentra en proporción al tamaño que presenta,

4) la "longitud" de distanciamiento o acortamiento de cada vínculo de relación debe traducirse en un "valor" (vectorial) con una escala de medida que reflejaría la calidad de relacionamiento existente entre un factor(es) y otro(s),

5) la "expansión" o, por el contrario, la "contracción" de los elementos alusivos a cada dimensión significa el camino hacia el cual transita cada factor, vale decir, si se dirige hacia un escenario "ideal", hacia uno "deseado", hacia uno "NO deseado", o hacia uno "incorrecto". ${ }^{3}$

Por último, 6) donde esa expresión tridimensional de la V.G. -que expone niveles de fortalecimiento o debilitamiento, niveles de relacionamiento, de posicionamiento, de expansión y/o contracción, direcciones hacia escenarios específicos en la dimensión espacio-tiempo, y niveles de deformación por influencia externa sobre los ejes de relaciones (entendido sobre lo que antes fue considerado como telaraña) o sobre uno o algunos factores de la V.G.- expone, en síntesis, también una resultante general que alude al "direccionamiento" general de toda la V.G. en la dimensión espacio-tiempo, vale decir, hacia cuál de los cuatro escenarios posibles ("ideal", "deseado", "NO deseado" o "incorrecto") se dirige en conjunto la Vulnerabilidad Global analizada en el tiempo.

${ }^{3}$ Este aspecto se tocará más adelante. Sin embargo, para profundizar sobre estos cuatro escenarios, se recomienda revisar Bolívar (2011: pp 203-212) 


\section{EL MÉTODO MULTI-VARIANTE EN ESCENA}

El método propone la identificación y selección de variables consideradas prioritarias por cada una de las ocho dimensiones. En esta investigación se planteó la identificación y selección de cinco variables por cada dimensión, asistidas cada una por tres indicadores. Siguiendo lo que plantea el método multi-variante, es importante señalar que lo que se valora son los niveles de "Vulnerabilidad", en otras palabras niveles de "debilidad", por lo que la mayor valoración que admite el método (10) es sinónimo de mayor vulnerabilidad, siendo 1 el menor grado de vulnerabilidad admitido.

El proceso de aplicación del método es básicamente "secuencial", es decir que la información producida en una etapa anterior se convierte en vital para el avance de una etapa posterior. No obstante, esto no quiere decir que su resolución es lineal, sino dialéctica, lo que permite la revisión constante de datos en retrospectiva, con el propósito de ajustar la información obtenida a través de los indicadores con fines verificativos o de actualización.

Planteamiento esquemático secuencial del método multi-variante:

- Identificación de (5) variables en cada dimensión de la V.G.

- Identificación de (3) indicadores en cada una de esas variables.

- Medición de indicadores (Detonadores, activadores y/o amenazas o vulnerabilidades internas y externas)

- Determinación (medición) del estado de cada factor de la V.G.

- Determinación del tipo y nivel de relacionamiento entre factores.

- Diagnóstico del estado de la V.G.

- Posibles derivaciones de cada factor en espacio-tiempo hacia los cuatro escenarios posibles.

- Posibles derivaciones de la V.G. en espaciotiempo hacia los cuatro escenarios posibles.

- Medidas y acciones preventivas para adelantarse a posibles caminos que puede tomar cada factor de la V.G.

Bajo ese esquema procedimental, seguidamente se presenta la caracterización de las dimensiones de la V.G. en Esquilán a través de la valoración de variables e indicadores.

\section{CARACTERIZACIÓN DE LAS DIMENSIONES DE LA VULNERABILIDAD GLOBAL Síntesis de resultados}

El área definida para la caracterización se concentró en 15 grandes manzanas definidas por el Instituto Nacional de Estadística, limitando al Norte por la vía férrea, al Sur por los Rio Rocha y Pampa Mayu, al Este por las calles "Ceres" e "Hinojos" y al Oeste por la Avenida El Testigo y torrentera Chijllawiri, con una población empadronada en 2012 de 1461 pobladores, que habitan en 401 viviendas censadas.

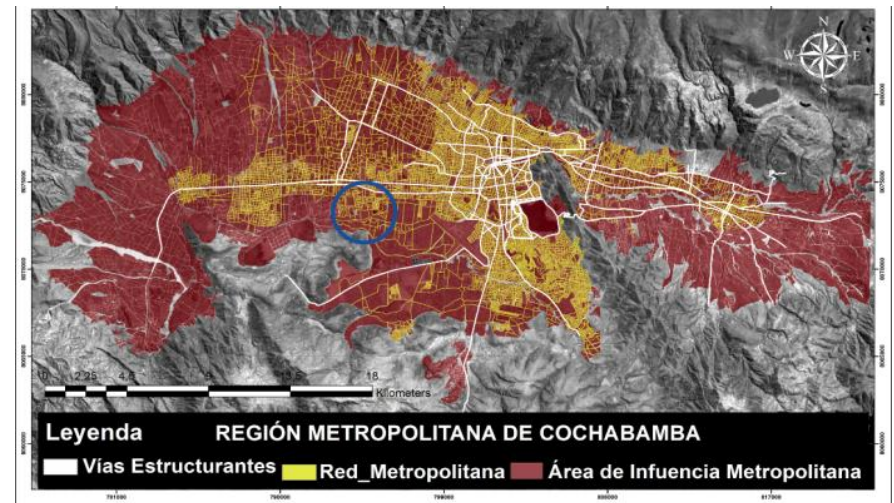

Gráfico N 14

Plano de ubicación con relación a la RMK

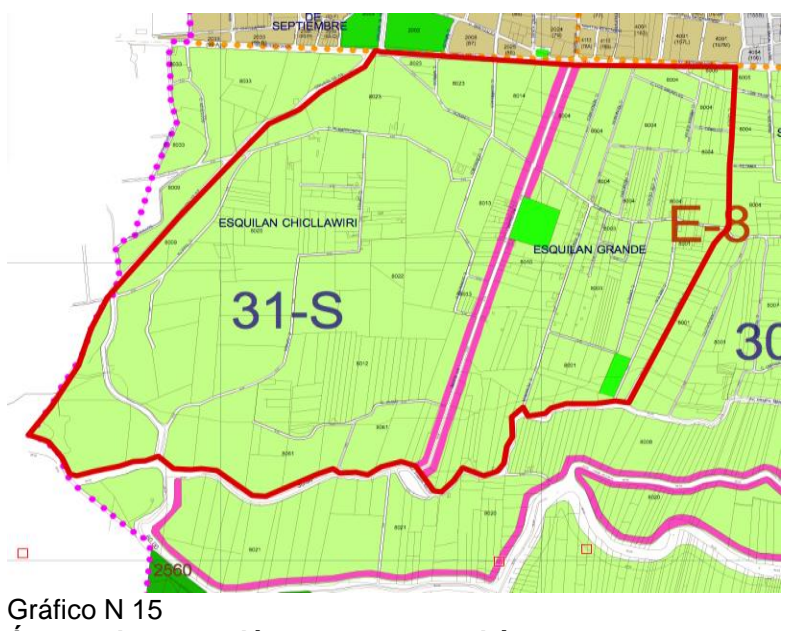

Área de intervención. Zona de Esquilán 
Importante: Debido a la extensión limitada de este artículo, se exponen a manera de ejemplo sólo dos cuadros de "variables" e "indicadores", correspondientes a las dimensiones Ecológica-ambiental y Física estructural. El análisis completo tanto de variables como indicadores de todas las dimensiones no se presenta en esta oportunidad. No obstante se debe mencionar que el análisis de todas las dimensiones fue exhaustivo por quien escribe este artículo durante la gestión 2016, bajo el amparo del Instituto de Investigaciones de Arquitectura de la Universidad Mayor de San Simón, y tuvo como resultado un análisis valorativo y comparativo de cada una de las variables e indicadores por cada Dimensión, computando 120 valoraciones en total.

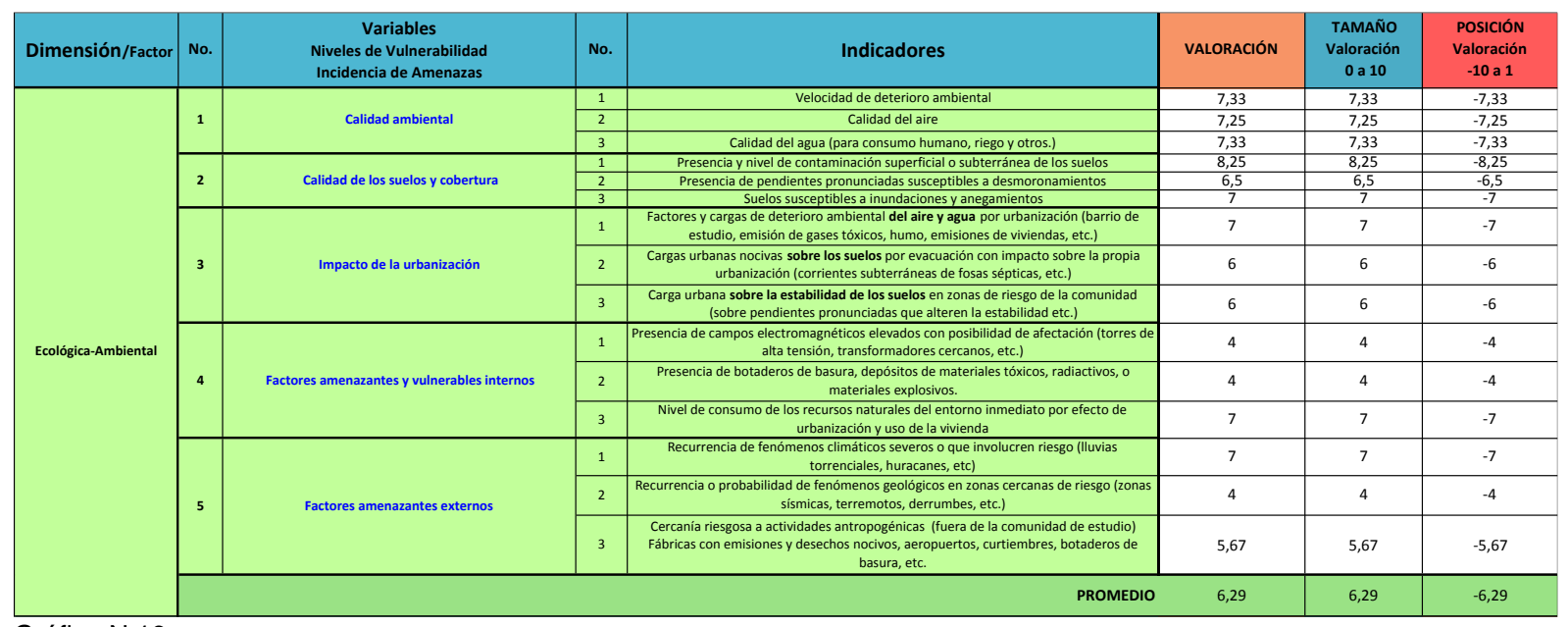

\section{Gráfico N 16}

Cuadro valorativo de la Dimensión Ecológica-Ambiental

Elaboración propia

\begin{tabular}{|c|c|c|c|c|c|c|c|}
\hline Dimensión/Factor & No. & $\begin{array}{l}\text { Variables } \\
\text { Niveles de Vulnerabilidad } \\
\text { Incidencia de Amenazas }\end{array}$ & No. & Indicadores & VALORACIÓN & $\begin{array}{l}\text { TAMAÑo } \\
\text { Valoración } \\
0 \text { a } 10\end{array}$ & $\begin{array}{l}\text { POSICión } \\
\text { Valoración } \\
-10 \text { a } 1\end{array}$ \\
\hline \multirow{16}{*}{ Fisica-Estructural } & \multirow{3}{*}{1} & \multirow{3}{*}{ Grado de exposición a factores de amenaza } & 1 & Cercanía de elementos vulnerables a focos de toxicidad y contaminación atmosférica & 8 & 8 & -8 \\
\hline & & & 2 & $\begin{array}{l}\text { Situación de elementos vulnerables con rel. a causes hídricos de riesgo, inundación y/o } \\
\text { anegamiento }\end{array}$ & 7 & 7 & -7 \\
\hline & & & 3 & $\begin{array}{l}\text { Situación de elementos vulnerables con relación a pendientes pronunciadas y suelos } \\
\text { inestables }\end{array}$ & 5 & 5 & -5 \\
\hline & \multirow{3}{*}{2} & \multirow{3}{*}{ Nivel de resistencia-Homeostasis } & 1 & $\begin{array}{c}\text { Calidad constructiva de las viviendas } \\
\end{array}$ & 6,75 & 6,75 & $-6,75$ \\
\hline & & & 2 & $\begin{array}{l}\text { Calidad y estado de la infraestructura básica (estado de calles, pendientes adecuadas, } \\
\text { estado de postes eléctricos, etc.). }\end{array}$ & 7 & 7 & -7 \\
\hline & & & 3 & $\begin{array}{l}\text { Calidad y estado de infraestructura especifica para resistir y hacer frente a las amenazas } y \\
\text { fenómenos que puedan presentarse. }\end{array}$ & 8,2 & 8,2 & $-8,2$ \\
\hline & \multirow{3}{*}{3} & \multirow{3}{*}{ Nivel de resistencia-Resiliencia } & 1 & $\begin{array}{l}\text { Disponibilidad de equipo y maquinaria para procesosos de "rehabilitación" de las } \\
\text { condiciones básicas }\end{array}$ & 8 & 8 & -8 \\
\hline & & & 2 & $\begin{array}{l}\text { Disponibilidad de equipo, maquinaria, mano de obray yaterial (insumos) para procesos } \\
\text { de "reconstrucción" básica }\end{array}$ & 6,2 & 6,2 & $-6,2$ \\
\hline & & & 3 & $\begin{array}{l}\text { Disponibilidad de insumos recursos humanos para e e restablecimiento de las } \\
\text { condiciones sociales, psicologicas y familiares de la comunidad }\end{array}$ & 9,5 & 9,5 & $-9,5$ \\
\hline & \multirow{3}{*}{4} & \multirow{3}{*}{ Nivel de Susceptibilidad } & 1 & $\begin{array}{l}\text { Cambios naturales en la estructura de los suelos en pendiente, en los cursos de agua, } \\
\text { desmoronamientos paulatinos de suelo que pueden tener efectos en cadena, etc. }\end{array}$ & 9 & 9 & -9 \\
\hline & & & 2 & $\begin{array}{l}\text { Intervenciones antrópicas en la comunidad que conlleven riesgo (movimiento de tierras, } \\
\text { construcciones irresponsables, etc.) }\end{array}$ & 10 & 10 & -10 \\
\hline & & & 3 & 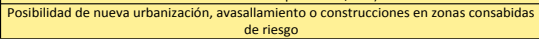 & 7,2 & 7,2 & $-7,2$ \\
\hline & \multirow{4}{*}{5} & \multirow{4}{*}{ Factores de Ubicación } & 1 & Ubicación física de viviendas con relación a infraestructura básica y de socorro & 9 & 9 & -9 \\
\hline & & & 2 & $\begin{array}{l}\text { Ubicación fisica de la infraestructura básica y de socorro con relación a factores de } \\
\text { amenaza }\end{array}$ & 5 & 5 & -5 \\
\hline & & & 3 & $\begin{array}{l}\text { Situación de la urbanización (comunidad) con relación a viás de escape e interconexiones } \\
\text { de ayuda en casos de desastre. }\end{array}$ & 7,2 & 7,2 & $-7,2$ \\
\hline & & & & PROMEDIO & 7,54 & 7,54 & $-7,54$ \\
\hline
\end{tabular}

\section{Gráfico N 17}

Cuadro valorativo de la Dimensión Física-Estructural

Elaboración propia

En ello, el siguiente cuadro representa una síntesis de resultados de valoración de Dimensiones, Variables e Indicadores, en las connotaciones de "valoración", "tamaño" y "posición" en el esquema espacio-tiempo, puntos que por razones de espacio se desarrollarán de manera sucinta en el apartado de conclusiones.

\begin{tabular}{|c|c|c|c|c|}
\hline DIMENSIÓN & VALORACIÓN & $\begin{array}{c}\text { TAMAÑO Valoración } \\
0 \text { a } 10\end{array}$ & $\begin{array}{l}\text { Factor gráfico de } \\
\text { reducción }(0,15)\end{array}$ & $\begin{array}{c}\text { POSICIÓN Valoración } \\
-10 \text { a } 1 \\
\end{array}$ \\
\hline Ecológica-Ambiental & 6,29 & 6,29 & 0,9435 & $-6,29$ \\
\hline Física-Estructural & 7,54 & 7,54 & 1,131 & $-7,54$ \\
\hline Económica & 4,73 & 4,73 & 0,7095 & $-4,73$ \\
\hline Técnica & 5,89 & 5,89 & 0,8835 & $-5,89$ \\
\hline Político-Institucional & 4,98 & 4,98 & 0,747 & $-4,98$ \\
\hline Educativa & 7,75 & 7,75 & 1,1625 & $-7,75$ \\
\hline Ideológica-Cultural & 7,23 & 7,23 & 1,0845 & $-7,23$ \\
\hline Social-Organizativa & 5,73 & 5,73 & 0,8595 & $-5,73$ \\
\hline
\end{tabular}


Gráfico N 18

Cuadro síntesis de valoración de variables e indicadores

Elaboración propia

Empero, ¿Cómo se representa la caracterización de la V.G. a través del examen gráfico-analítico?

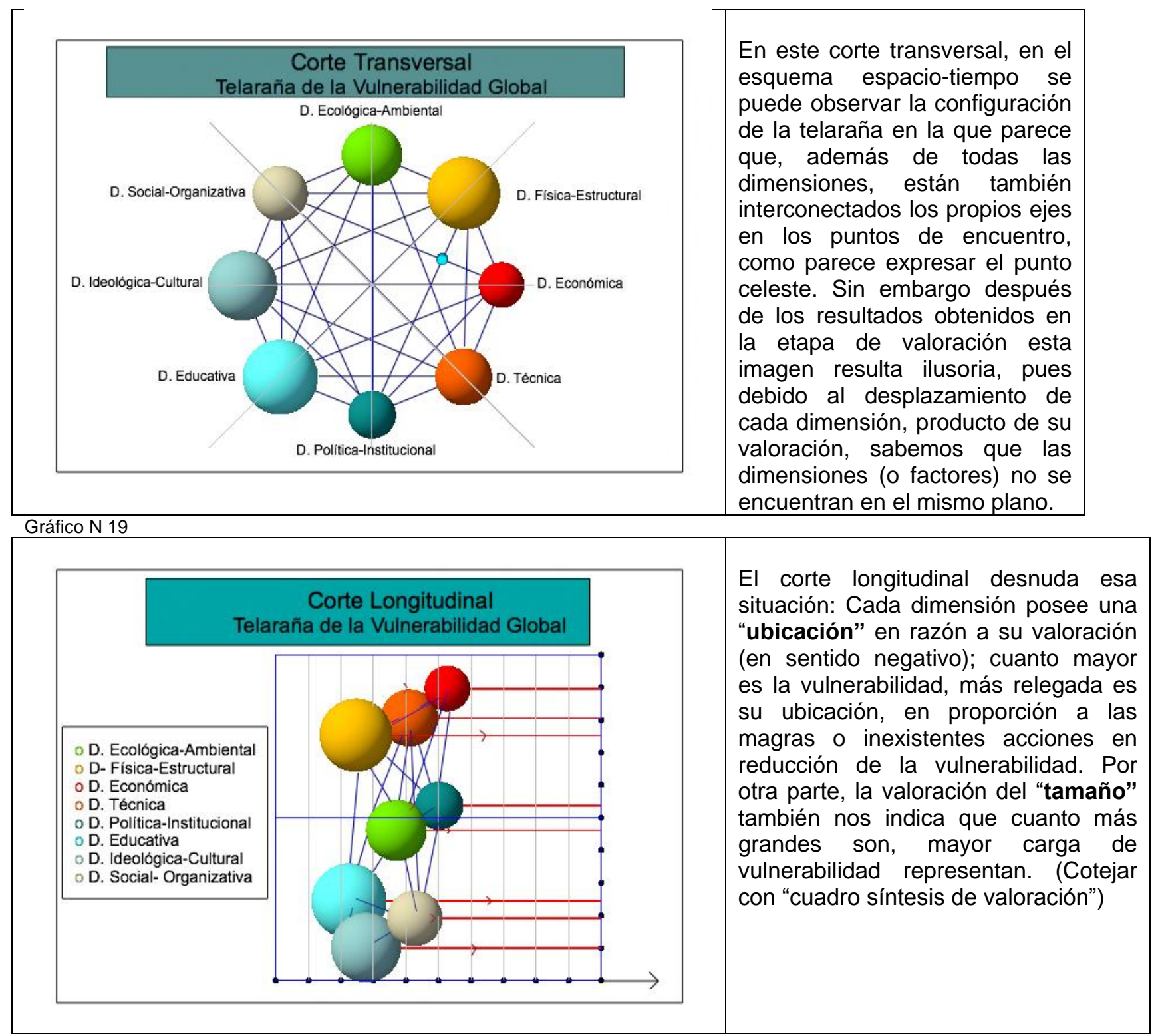

Gráfico N 20 


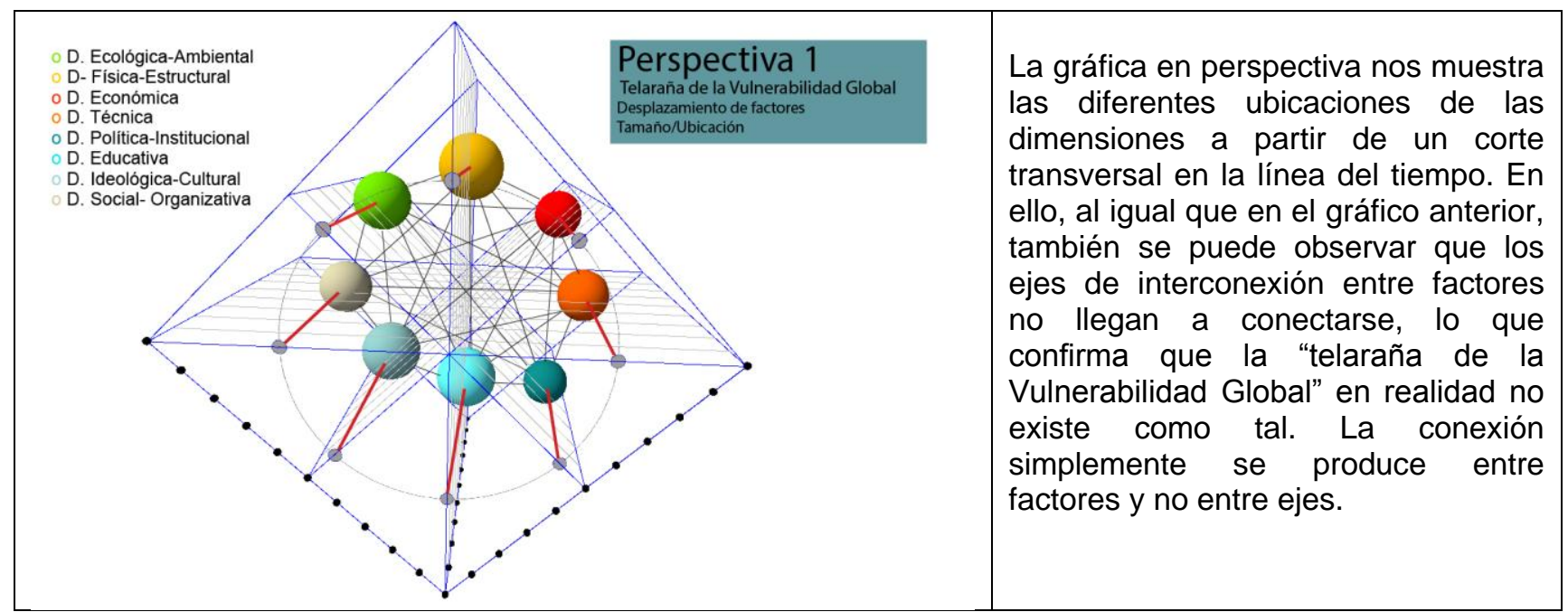

\section{Gráfico N 21}

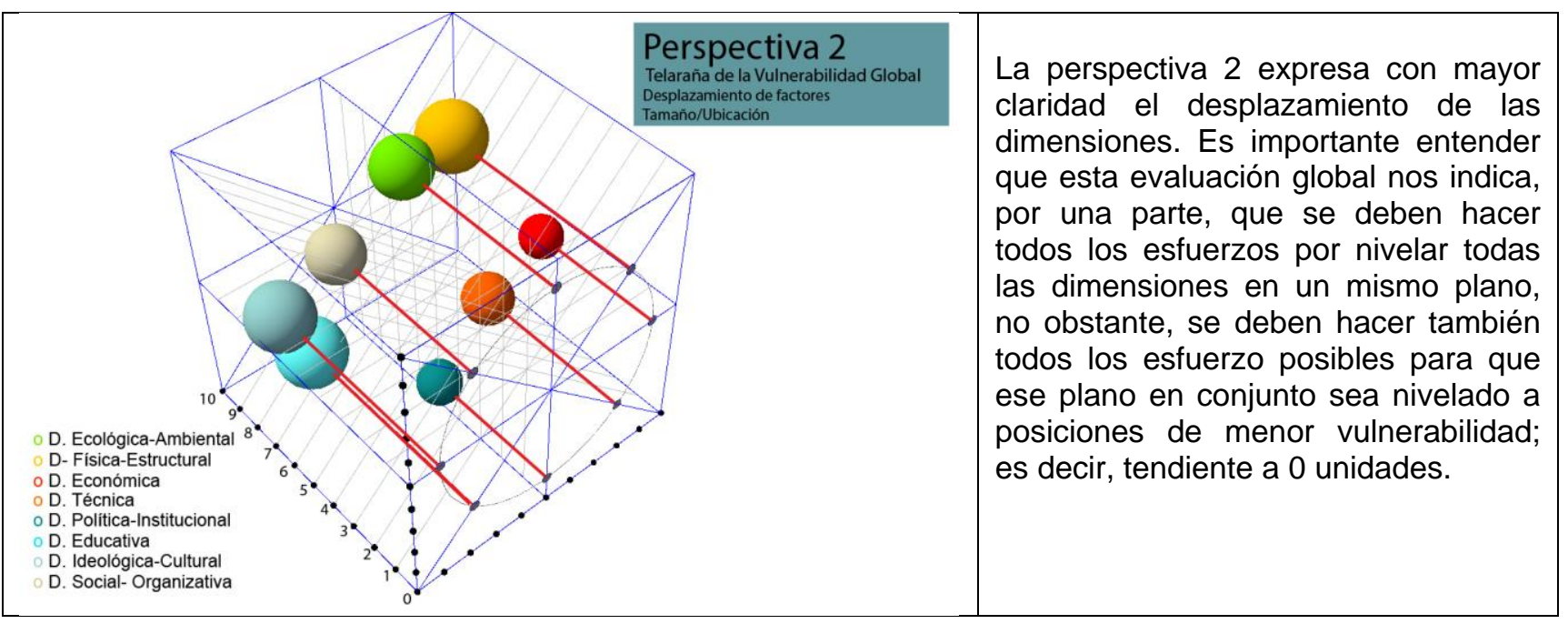

Gráfico N 22

\subsection{Longitud}

La "longitud" entre dimensiones alude a la distancia existente entre cada una de ellas traducidas en un "valor vectorial". Si nos remitimos al Corte longitudinal o a la Perspectiva 1, podremos observar que las distancias entre dimensiones no son las mismas y que su variación debe partir de la valoración de "longitud", que será la variación vectorial final expresada en porcentaje cuando el ejercicio de la modelación tridimensional haya culminado. No debemos perder de vista que la diferencia gráfica entre factores que exhibe la telaraña es sólo gráfica, y que los valores de "longitud" entre factores en realidad corresponde a una relación medida en porcentaje en el espacio-tiempo.

De todo ese análisis se pudo diagnosticar que las dimensiones "Ecológica-Ambiental", "Física-Estructural", "Educativa" e "Ideológica-Cultural" son las que presentan mayor vulnerabilidad. El tamaño que representan y su ubicación emplazan a todos los actores involucrados a interactuar en la reducción de las mismas. La práctica de este ejercicio analítico que propone el método sirvió para identificar en primera instancia a los distintos actores que intervienen en el problema tanto como las deficiencias en el rol que desempeñan; en ello será importante a futuro trabajar primero en la nivelación (tendiente a 0) de todos los indicadores, para de esta manera tratar de nivelar todas las variables y, por supuesto, todas las dimensiones en un mismo plano que tienda a la valoración 0 . Tarea ardua tanto para las instituciones involucradas como para la sociedad, que deberá concentrarse en reducir el factor de "longitud" entre cada una de las dimensiones. 
La valoración de la Vulnerabilidad Global a través del método diagnosticó también que el nivel de compromiso de cada dimensión en la solución del problema no es integral. Si bien las dimensiones "Económica" y "Político-Institucional" presentaron las menores valoraciones de vulnerabilidad intrínseca -lo que es positivo-, esta situación debió también proyectarse hacia otras dimensiones; esto quiere decir, acortar las distancias de "longitud" entre dimensiones. La relativa fortaleza que ostentan intrínsecamente estas dos dimensiones (en la medida de la evaluación realizada) no es suficiente en el esquema de la Vulnerabilidad Global pues su condición no ayuda en gran medida al restablecimiento de las dimensiones con mayor vulnerabilidad.

\subsection{Resistencia}

Resultó importante también diagnosticar los niveles de "resistencia" entre conexiones. En forma paralela a lo que esboza el escenario de relaciones diferenciadas por "longitud" entre los distintos factores de la Vulnerabilidad Global (información gráfica y matemática), los resultados del análisis de indicadores también arrojaron elementos de valoración cualitativa que dieron cuenta sobre la "resistencia" de los ejes de relación. Esta información alude tanto a aspectos positivos como negativos que deben ser valorados para determinar también potencialidades o amenazas en las relaciones entre factores.

Luego de un análisis juicioso, se pudo evidenciar diferencias cualitativas en el sistema de relaciones de conexión que se expresan en el siguiente gráfico:

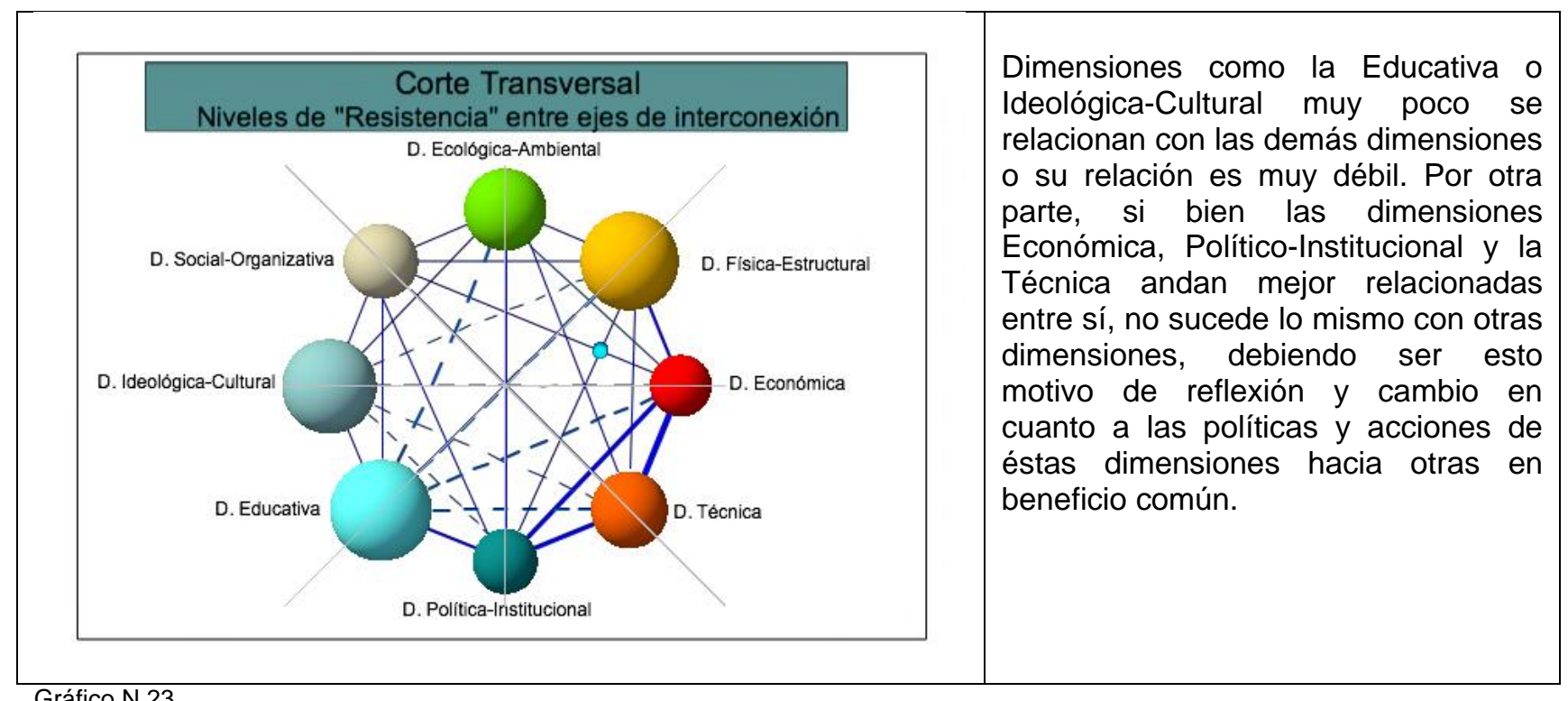

Gráfico N 23

\subsection{Conexión}

Uno de los beneficios de la modelación tridimensional es que permite observar en espacio-tiempo la posibilidad o no de conexión entre los ejes que relacionan a los factores, es decir entre los ejes que en realidad debieran conformar la telaraña.

Para medir esa situación se debe seguir el mismo camino metodológico que el de la obtención del valor vectorial de "longitud". Sin embargo esta tarea es realmente ardua desde la metodología gráfica-matemática que el método propone, debido a la gran cantidad de puntos que debieran medirse. Empero su ejercicio con propósitos específicos entre factores también específicos resulta muy importante para aquellos fines de "proxies" o seguimiento que advierte Omar Darío Cardona (2007). Medir la separación entre los ejes de relación con fines analíticos permitiría conocer sobre los esfuerzos conjuntos que todos los factores realizan con el propósito de alcanzar la conexión de la telaraña; en síntesis, menor Vulnerabilidad Global o mayor resistencia Global (Bolívar, 2015).

En el análisis se vio que el trabajar en la reducción de la vulnerabilidad en las dimensiones "FísicaEstructural", "Educativa" e "Ideológica-Cultural" sería un equivalente a estrechar los vínculos de "Iongitud" entre dimensiones, por una parte, pero principalmente estrechar los espacios existentes entre ejes de conexión; en otras palabras, generar la posibilidad de conformación de una telaraña robusta de la 
Vulnerabilidad Global. Remitiéndonos al esquema espacio-tiempo vemos que si se bajan los niveles de vulnerabilidad en las dimensiones "Física-Estructural" y "Educativa", es decir, si se reducen sus "tamaños" y si se nivelan sus "ubicaciones" tendiendo a 0 , el eje de interconexión entre estas dos dimensiones también se acercará a 0 , logrando con esto llegar a juntarse el eje de relación con otros ejes que interconectan otras dimensiones; las dimensiones "Ecológica-Ambiental" y "Política-Institucional", a manera de ejemplo.

De esa manera, el método multi-variante promueve gráfica, matemática y espacialmente revisar a nivel diagnóstico las políticas y acciones que se realizan (o no, por omisión) a favor de la reducción de la vulnerabilidad. Por otra parte, promueve también el análisis prospectivo de las posibles políticas y acciones que debieran realizarse para tratar de nivelar todos las dimensiones en un mismo plano que tienda a la valoración 0 en el esquema espacio-tiempo.

\section{POSIBLES CAMINOS DINÁMICOS DE LA VULNERABILIDAD GLOBAL POR LOS QUE PODRÍA TRANSITAR LA V.G. EN ESQUILÁN.}

En anteriores investigaciones habíamos propuesto el concepto "transformación cualitativa de la vulnerabilidad" y la noción de la "relación dinámica e interactuante entre amenazas y vulnerabilidades". Se vio que tanto ese concepto como esa relación se desarrollan dinámicamente en la "dimensión espaciotiempo" y que la vulnerabilidad (alimentada también por esa relación interactuante con la amenaza) podía transformarse cualitativamente en el tiempo y dirigirse hacia cuatro escenarios posibles: hacia un escenario "ideal" (donde tanto las vulnerabilidades como lo impactos de los fenómenos sean 0), hacia uno "deseado" (donde a pesar de la manifestación de los fenómenos la vulnerabilidad sea 0), hacia uno "no deseado" (donde tanto la vulnerabilidad como los fenómenos sean altos y severos), o hacia uno "incorrecto" (donde a sabiendas que la intensidad de los fenómenos son 0 o mínimos se deje crecer la vulnerabilidad a niveles extremos).

Por una parte, las vulnerabilidades de cada dimensión podrían estar transformándose cualitativamente a través del tiempo, lo que confirma la necesidad de actualizar constantemente los valores de variables e indicadores. Por otra parte, en el esquema espacio-tiempo cada dimensión de la Vulnerabilidad Global podría estar derivando en caminos distintos; hacia escenarios "incorrectos", dependiendo su cualidad (física, educativa, social, etc.) o hacia escenarios "no deseados". En ese esquema, la telaraña de la Vulnerabilidad Global tendería a deformarse aún más y los esfuerzos por recomponer la misma serían mucho más arduos.

De todo ello entonces es imperante considerar tres aspectos: (1) Algunas variables (condición intrínseca, amenazas al fin) podrán definir o agravar la vulnerabilidad por su debilitamiento intrínseco (de origen interno), (2) algunas variables (amenazas de origen externo) podrán simplemente alimentar o afectar la vulnerabilidad y (3) algunas variables (amenazas externas en efecto dinámico con las condiciones intrínsecas de cada vulnerabilidad) podrán retroalimentar la vulnerabilidad por efecto cíclico (caso la informalidad urbanística). De ello convenimos entonces que algunas Amenazas (o variables, en su caso) podrán no sólo afectar a una dimensión (o factor de la V.G.), sino a otros factores.

Resulta importante, entonces, que la valoración prospectiva se realice individualmente por cada dimensión, esquematizando escenarios posibles a futuro que deriven de las acciones técnicas, sociales, políticas, educativas, etc. Los resultados de esa evaluación prospectiva permitiría el adelantamiento de posibles sucesos negativos y el posicionamiento hacia escenarios más "deseados", para luego establecer mejores relaciones de interconexión entre las demás dimensiones.

En el caso de Esquilán, los posibles caminos dinámicos sugieren que si no se trabaja en la reducción de la vulnerabilidad Educativa, a través de planes y programas educativos efectivos en Gestión de Riesgos, simulacros, mesas redondas entre padres de familia, profesores, autoridades y estudiantes, en la actualización de sistemas de prevención y otros, la deformación de la telaraña en el tiempo puede ser más riesgosa de lo que se evidencia en la actualidad. En paralelo, resultará importante también trabajar en mejorar las relaciones de la dimensión Educativa con la Dimensión Ideológica-Cultural, pues el cambio de percepción externa y de actitud pasiva con relación a los riesgos debe lograrse a través del relacionamiento con otras dimensiones. 


\section{REFLEXIONES A MANERA DE CONCLUSIÓN}

De la puesta en práctica del método, en cuanto a la valoración de variables e indicadores, hemos aprendido que -dependiendo el escenario de estudio y sus problemas intrínsecos- muchos de los promedios finales por cada variable y cada dimensión pueden resultar por lo general cercanos a la media de cada problema, debido a que algunas vulnerabilidades altas se compensan con la poca vulnerabilidad manifestada en otros indicadores. En ello es importante reflexionar principalmente sobre aquellos indicadores que marcan vulnerabilidades elevadas, pues la sola presencia de esa vulnerabilidad puede ser altamente nociva para la población, produciendo riesgos elevados susceptibles a derivar en desastres de magnitud. A manera de ejemplo, si bien la pésima calidad del aire puede darse con mayor intensidad en algunos sectores de Esquilán más que en otros, las enfermedades respiratorias y gastrointestinales que puedan generarse en la zona de mayor riesgo son propensas a transmitirse por contagio o transportación de vectores hacia los sectores donde la calidad del aire es más salubre. En síntesis, no sólo se debe trabajar en reducir los índices de vulnerabilidad en cada variable o dimensión de manera general (de manera compensada), sino ser enfáticos en reducir principalmente aquellas vulnerabilidades puntuales cuyos indicadores muestran índices elevados y no permanecer en la comodidad de equilibrar promedios con vulnerabilidades cuyos indicadores exponen menor vulnerabilidad; lo ideal, desde lo que persigue el método, es equilibrar los índices de vulnerabilidad tendiendo a 0 , es decir reduciendo la vulnerabilidad en todos los problemas identificados, para así tender a unificar todas la dimensiones en un solo plano que tienda también a la ubicación 0 , lo que sería un escenario ideal.

De esta experiencia se ha confirmado que medir la vulnerabilidad y más aún si esta es medida en su condición "global", resulta una tarea de complejidad. Sin embargo su aproximación de manera más certera puede ayudarnos a los fines de proxies o seguimiento para trabajar prospectivamente sobre posibles escenarios futuros. Para ello, habrá que considerar varios puntos que deberán ser vencidos en la medida de lo posible:

1. Es crucial entender sin equívocos que la vulnerabilidad está compuesta por la interacción de diversos factores (dimensiones) que hacen a la vulnerabilidad global, por lo que medir solamente un factor sería tan sólo un acto parcial del problema. La medición de la vulnerabilidad debe ser integral (global), pues esta se retroalimenta entre todos sus factores o dimensiones.

2. La vulnerabilidad no debe ser entendida sólo como un concepto, sino el compendio de varias situaciones reales caracterizadas por (a) el "grado de exposición" y (b) los "niveles de resistencia", compuesto por la "homeostasis" (protección y reacción inmediata) y la "resiliencia" (recuperación básica y reconstrucción), por lo que cada dimensión de la vulnerabilidad presentará también un compendio de todos esos componentes.

3. Este compendio de varias situaciones podrá manifestarse distinto en cada dimensión de la vulnerabilidad. A manera de ejemplo, la "protección" en la dimensión física-estructural podrá referirse precisamente a los niveles de seguridad y calidad en las construcciones, así como en la calidad de las intervenciones de prevención en el contexto físico-estructural. No obstante, podrán existir también niveles de "protección" en la dimensión política, precisamente a través del planteamiento y acciones políticas dirigidas a establecer prácticas de prevención así como partidas económicas específicas para temas de prevención. De igual forma, la "protección" en la dimensión educativa tendrá otros parámetros de valoración referidos a programa educativos dirigidos a conocer, mejorar y practicar niveles de prevención que irán (o deberían ir), de manera recíproca y articulada, a fortalecer las prácticas constructivas de parte de las familias en la dimensión física, a fortalecer y enfocar demandas ciudadanas en cuanto a políticas públicas se refiere, etc. Este panorama sucedería con todas las dimensiones de la vulnerabilidad global y con cada uno de los componentes que definen a la vulnerabilidad (Grado de Exposición y Niveles de Resistencia-junto con todos sus otros componentes), situación que pone mayores grados de dificultad a su medición y que habrá que resolver enfocando una escala de trabajo pertinente y variables objetivas que puedan ser trabajables en el diseño y aplicación del método.

4. Otra dificultad que se deberá vencer está referida a los aspectos "tangibles e "intangibles" de la vulnerabilidad, pues, a manera de ejemplo, resulta que aspectos (componentes) de la vulnerabilidad en la dimensión física pueden ser cuantificables tangiblemente precisamente desde sus aspectos físicos, sin embargo en la dimensión cultural o educativa, muchos de los aspectos inherentes a la vulnerabilidad 
deberán ser cualificados en una atmósfera de parámetros intangibles. La correcta definición y la precisa clasificación de los componentes (tangibles o intangibles) permitirán resultados más certeros.

5. Por otra parte, cada dimensión de la vulnerabilidad estará condicionada por diversas variables: estas variables podrán ser "externas" o "intrínsecas" a la vulnerabilidad, sin embargo ambas con capacidad de afectar a la vulnerabilidad. Las variables "externas" podrán considerarse (en su caso) como "amenazas", capaces de alterar por efecto cíclico (explicado con anterioridad) los diversos componentes de la vulnerabilidad (Grado de exposición y Resistencia); es decir que la vulnerabilidad dependería en gran medida de la magnitud de la amenaza. Por otra parte, las variables "intrínsecas" definirían el estado inicial de los componentes de la vulnerabilidad; vale decir la condición "intrínseca" que define el estado inicial de los componentes de la propia vulnerabilidad.

6. En el caso de que la(s) variable(s) de análisis fuera(n) una amenaza, podríamos entonces formular hipotéticamente que al juntarse cada "dimensión" con la "amenaza" que la incrementa se formaría un micro "escenario de riesgo" (amenaza X vulnerabilidad), por lo que un paso importante para reducir la vulnerabilidad podría empezar por reducir las variables "externas", en otras palabras las "'amenazas", a tiempo de reducir las variables "intrínsecas" (o vulnerabilidades internas) que condicionan los niveles de debilidad en la estructura misma de la vulnerabilidad, es decir en sus componentes.

7. En ello será importante establecer que (a) algunas "amenazas" podrán retroalimentar la vulnerabilidad (como la amenaza de la informalidad urbanística en la dimensión física-estructural) y viceversa; (b) otras "amenazas" (externas) podrán simplemente alimentar o afectar la vulnerabilidad; y (c) otras "variables" (internas) podrán agravar la condición de la vulnerabilidad por su debilitamiento intrínseco. Resultará importante entonces considerar y clasificar el o los tipos de variables que afecten a cada dimensión de la vulnerabilidad.

8. Con relación a este punto, será vital también considerar que una o muchas variables relacionadas con una dimensión podrán afectar indirectamente a otras dimensiones de la vulnerabilidad global. En qué medida y cómo, es otra dificultad que se debe superar. Asimismo, habrá que pensar también que la clasificación de las variables e indicadores deberán considerar si la variable corresponde a un nivel de estado de "resistencia" (ex ante) o resiliencia (ex post).

Por último, es bueno recordar que el método expone su capacidad prospectiva al confirmar también que las valoraciones positivas en los indicadores no sólo deben contribuir al fortalecimiento de cada dimensión, sino también contribuir al fortalecimiento de otras dimensiones. De ahí la responsabilidad de quienes intervienen en cada dimensión por trazar políticas integrales, visiones colectivas, acciones conjuntas, inversiones encadenadas, etc. El método promueve la idea que toda dimensión, más allá de su cualidad intrínseca tendiente a lo ambiental, a lo físico, a lo económico, educativo, etc., debiera considerar precisamente la integralidad, la colectividad, la conjunción y el encadenamiento positivo en todas las acciones de planificación y ejecución; desde lo individual, grupal y colectivo.

\section{BIBLIOGRAFÍA}

BLAIKIE, P., CANNON, T., DAVIS, I y WISNER, B. (1996) "Vulnerabilidad. El entorno social, político y económico de los desastres". Bogotá-Colombia. La Red. ITDG.

BOLIVAR, H. (2011) "Suelo Urbano, Vulnerabilidad y Riesgo de Desastres", ASDI-DICyT-UMSS, Ed. Poligraf, Cochabamba, Bolivia. (2013) "La Urbanización de los Riesgos: Camino a la in-sustentabilidad urbana". In URBimetría. Revista Boliviana de Estudios del Hábitat. Vol 1 No1. Instituto de Investigaciones de Arquitectura y Ciencias del Hábitat". Universidad Mayor de San Simón. Editorial Anthropos. Cochabamba, Bolivia.

(2015) "Metodología multivariante para la medición de la vulnerabilidad". Instituto de Investigaciones de Arquitectura. Universidad Mayor de San Simón (sin publicar), Cochabamba, Bolivia.

CARDONA, O. D. (1993) "Evaluación de la Amenaza, la Vulnerabilidad y el Riesgo. Elementos para el Ordenamiento y la Planeación del Territorio", en MASKREY, Andrew. "Los Desastres No Son Naturales", LA RED.

(2007) "Midiendo lo Inmedible: Indicadores de Vulnerabilidad y Riesgo". (en línea) Disponible en:

(http://www.desenredando.org/public/omar) (25-09-2007)

(http://www.structuralia.com/info/img//Noticia_Ficheros/Midiendo\%20lo\%20inmedible.pdf) (01/10). 
(http://www.idrc.ca/uploads/user-S/114907933016_cardona_esp.pdf) (03-11-10)

CLICHEVSKY, N. (2000) "Informalidad y Segregación Urbana en América Latina. Una aproximación". Consejo Nacional de Investigaciones Científicas y Técnicas-Universidad de Buenos Aires, CONICETUBA. Buenos Aires.

LAVELL, A. (1996) "Degradación Ambiental, Riesgo y Desastre Urbano. Problemas y Conceptos: Hacia la definición de una agenda de investigación, en FERNANDEZ, María Augusta. (Compiladora). "Ciudades en Riesgo": Degradación Ambiental, Riesgos Urbanos y Desastres. LA RED.

PRADO, P. (2006) "Crecimiento Urbano del Área Metropolitana de Cochabamba, Escenarios para el Futuro". Universidad Mayor de San Simón, Facultad de Arquitectura, IIA - Progeo. Cochabamba, Bolivia.

VARGAS, J. E. (2002) "Políticas públicas para la reducción de la vulnerabilidad frente a los desastres naturales y socio-naturales". Serie: Medioambiente y Desarrollo № 50 . División de Desarrollo Sostenible y Asentamientos Humanos. Publicación de las Naciones Unidas y la CEPAL/ECLAC, Santiago de Chile.

VEYRET, Y. (2007) "Os Riscos: O homem como agressor e vítima do meio ambiente". Tradução do original: "Les risques" (2003). Editora Contexto. São Paulo, Brasil.

WILCHES-CHAUX, G. (1993) "La vulnerabilidad Global". En MASKREY, Andrew (Ed) "Los Desastres no son Naturales". La RED. Tercer Mundo Editores. Colombia.

. (1998) "Auge, caída y levantada de Felipe Pinillo, Mecánico y Soldador, o Yo voy a correr el riesgo". Publicado por LA RED, Red de Estudios Sociales en Prevención de Desastres en América Latina. Editorial e Imprenta DELTA S. C. Quito-Ecuador. (2007) “¿Qu-ENOS pasa?”: Guía de LA RED para la Gestión Radical de riesgos Asociados con el Fenómeno ENOS. IAI-LA RED. ARFO Editores e Impresores Ltda. Bogotá-Colombia.

\section{Bibliografía de apoyo}

Plan de Desarrollo Municipal del Municipio de Colcapirhua PDM. (2005)

Plan de Desarrollo Municipal del Municipio de Colcapirhua PDM. (2009)

Plan Urbano del Municipio de Colcapirhua. Gestión 2003-2013

Diagnóstico Integral Participativo del Municipio de Colcapirhua (2009) 